\title{
Low-Complexity MIMO Precoding for Finite-Alphabet Signals
}

\author{
Yongpeng Wu, Chao-Kai Wen, Derrick Wing Kwan Ng, \\ Robert Schober, and Angel Lozano
}

\begin{abstract}
This paper investigates the design of precoders for single-user multiple-input multiple-output (MIMO) channels, and in particular for finite-alphabet signals. Based on an asymptotic expression for the mutual information of channels exhibiting line-of-sight components and rather general antenna correlations, precoding structures that decompose the general channel into a set of parallel subchannel pairs are proposed. Then, a low-complexity iterative algorithm is devised to maximize the sum mutual information of all pairs. The proposed algorithm significantly reduces the computational load of existing approaches with only minimal loss in performance. The complexity savings increase with the number of transmit antennas and with the cardinality of the signal alphabet, making it possible to support values thereof that were unmanageable with existing solutions. Most importantly, the proposed solution does not require instantaneous channel state information (CSI) at the transmitter, but only statistical CSI.
\end{abstract}

This paper was presented in part at IEEE ICC 2016.

Y. Wu is with Institute for Communications Engineering, Technical University of Munich, Theresienstrasse 90, D-80333 Munich, Germany (Email:yongpeng.wu2016@gmail.com). The work of Y. Wu is supported by TUM University Foundation Fellowship.

D. W. K. Ng is with the School of Electrical Engineering and Telecommunications, University of New South Wales, Sydney, N.S.W., Australia (E-mail: w.k.ng@unsw.edu.au).

C. K. Wen is with the Institute of Communications Engineering, National Sun Yat-sen University, Kaohsiung 804, Taiwan (Email: chaokai.wen@mail.nsysu.edu.tw).

R. Schober is with the Institute for Digital Communications, Universität Erlangen-Nürnberg, Cauerstrasse 7, D-91058 Erlangen, Germany (Email: robert.schober@fau.de).

A. Lozano is with Universitat Pompeu Fabra, 08018, Barcelona, Spain (Email: angel.lozano@upf.edu). His work is supported by Project TEC2015-66228-P (MINECO/FEDER, UE) and by the European Research Council under the H2020 Framework Programme/ERC grant agreement 694974 


\section{INTRODUCTION}

Although complex Gaussian signals are capacity-achieving under perfect channel state information (CSI) at the receiver, signals conforming to discrete constellations are transmitted in practice. For such signals, the capacity-achieving approach - allocating more power to stronger channels — can be quite suboptimal, as illustrated for parallel channels in [1] and [2], and hence it is of interest to devise suitable precoders.

For multiple-input multiple-output (MIMO) channels with instantaneous CSI at the transmitter, an optimal linear precoder design was put forth in [3], building upon earlier works [4]-[8]. In turn, [9] proposed to group MIMO subchannels in pairs and design the relevant parameters within each pair and among pairs to increase the mutual information with finite-alphabet signals. This significantly reduces the complexity of the precoder optimization with little loss in mutual information. Recently, this idea was extended to pair multiple subchannels on the basis of a per-group precoding (PGP) technique [10], [11]. As an alternative way of reducing the computational load, a precoder design that optimizes a lower bound of the mutual information (rather than the actual mutual information) was set forth in [12].

With only statistical CSI available at the transmitter, the MIMO precoding design for capacity-achieving Gaussian signals was addressed in [13]-[19]. For discrete signals, an iterative precoding algorithm was proposed in [20] for the Kronecker channel model, yet the complexity of this complete-search algorithm is exponential in the number of transmit antennas and, even with modest numbers thereof (say, eight), it becomes unmanageable.

The premise of instantaneous CSI at the transmitter is reasonable when users are static or slowly moving, such that the fading remains constant for a sufficiently long time. With fast moving users, a more appropriate premise is to consider only statistical CSI at the transmitter. This paper proposes a low-complexity precoder design for rather general single-user MIMO channels with finite-alphabet inputs and statistical CSI at the transmitter. The contributions of the paper are as follows.

1) An asymptotic (in the number of antennas) expression is derived for the mutual information of MIMO channels with finite alphabet inputs and correlated Rice fading.

2) By exploiting the spatial characteristics of the adopted MIMO channel model, the left singular matrix of the optimal precoder is obtained. For positive Rice factors, the result differs from the one obtained in [20] for the Kronecker channel model.

3) Structures are established for the power allocation matrix and the right singular matrix of the pre- 
coder. These structures decouple the data streams over parallel equivalent subchannels, eliminating the need for a complete search of the entire signal space during the precoder optimization. The complexity of such optimization is thereby reduced by an exponential order of magnitude.

4) A novel low-complexity iterative algorithm is devised for the precoder optimization. This algorithm drastically reduces the computational load, but with only minimal loss-established on the basis of the 3GPP spatial channel model (SCM) [21]—in performance.

5) Some special cases are investigated, chiefly massive MIMO where the additional structure in the channel simplifies the derived algorithm.

The remainder of the paper is organized as follows. Section II briefly introduces some notation and describes the channel model. In Section III, the complete-search algorithm is reviewed and an idea proposed for reducing its computational complexity. Building on this idea, Section IV proposes a lowcomplexity precoding approach. Numerical results are provided in Section V, and conclusions are drawn in Section VI.

\section{PRELIMINARIES}

\section{A. Notation}

The following notation is adopted throughout: Superscripts $(\cdot)^{T},(\cdot)^{*}$, and $(\cdot)^{H}$ stand for the matrix/vector transpose, conjugate, and conjugate-transpose operations, respectively, $\operatorname{diag}\{\mathbf{b}\}$ denotes a diagonal matrix containing the entries of vector $\mathbf{b}, \operatorname{diag}\{\mathbf{B}\}$ denotes a diagonal matrix containing in the main diagonal the diagonal elements of matrix $\mathbf{B}, \odot$ and $\otimes$ denote the entry-wise and the Kronecker product of two matrices, respectively, $\operatorname{vec}(\mathbf{A})$ is a column vector containing the stacked columns of matrix $\mathbf{A},[\mathbf{A}]_{m n}$ denotes the $(m, n)$ th entry of matrix $\mathbf{A},[\mathbf{a}]_{m}$ denotes the $m$ th entry of vector $\mathbf{a},\|\cdot\|_{F}$ denotes the Frobenius norm, and $E[\cdot]$ represents the expectation with respect to the random variable inside [.], which can be a scalar, vector, or matrix. Finally, DA denotes the integral measure for the real and imaginary parts of the entries of A. That is, for an $n \times m$ matrix $\mathbf{A}$,

$$
D \mathbf{A}=\prod_{i=1}^{n} \prod_{j=1}^{m} \frac{d \operatorname{Re}[\mathbf{A}]_{i j} d \operatorname{Im}[\mathbf{A}]_{i j}}{\pi}
$$

where $\operatorname{Re}[\cdot]$ and $\operatorname{Im}[\cdot]$ return the real and imaginary parts, respectively. 


\section{B. Channel Model}

Consider a single-user MIMO channel where a transmitter and a receiver are equipped with $N_{\mathrm{t}}$ and $N_{\mathrm{r}}$ antennas, respectively. The received signal $\mathbf{y} \in \mathbb{C}^{N_{\mathrm{r}}}$ can be written as

$$
\mathbf{y}=\mathbf{H x}+\mathbf{n}
$$

where $\mathbf{H} \in \mathbb{C}^{N_{\mathrm{r}} \times N_{\mathrm{t}}}$ is a random channel matrix whose $(i, j)$ th entry denotes the complex fading coefficient between the $j$ th transmit and the $i$ th receive antenna1, $\mathbf{x} \in \mathbb{C}^{N_{\mathrm{t}}}$ denotes the zero-mean transmitted vector with covariance matrix $\Sigma_{\mathbf{x}}$, and $\mathbf{n} \in \mathbb{C}^{N_{\mathrm{r}}}$ is the zero-mean complex Gaussian noise vector with covariance matrix $\mathbf{I}_{N_{\mathrm{r}}}$. The transmit vector $\mathrm{x}$ satisfies the power constraint

$$
\operatorname{tr}\left(\boldsymbol{\Sigma}_{\mathbf{x}}\right) \leq P
$$

Based on the available CSI, and subject to the power constraint, we want to optimize $\Sigma_{\mathrm{x}}$ to maximize the spectral efficiency.

\section{Precoder Designs for Single-User MiMO Channel}

Let $\mathbf{x}=\mathbf{B} \mathbf{d}$, where $\mathbf{B} \in \mathbb{C}^{N_{\mathrm{t}} \times N_{\mathrm{t}}}$ is the precoder whereas $\mathbf{d} \in \mathbb{C}^{N_{\mathrm{t}} \times 1}$ is a signal vector whose entries are drawn independently from an equiprobable $M$-ary constellation; there are $M^{N_{\mathrm{t}}}$ possible signal vectors, the $m$ th of which is denoted by $\mathbf{d}_{m}$. The precoder admits the singular value decomposition (SVD) $\mathbf{B}=\mathbf{U}_{\mathbf{B}} \boldsymbol{\Lambda}_{\mathbf{B}} \mathbf{V}_{\mathbf{B}}$ where $\boldsymbol{\Lambda}_{\mathbf{B}} \in \mathbb{C}^{N_{\mathrm{t}} \times N_{\mathrm{t}}}$ is diagonal while $\mathbf{U}_{\mathbf{B}} \in \mathbb{C}^{N_{\mathrm{t}} \times N_{\mathrm{t}}}$ and $\mathbf{V}_{\mathbf{B}} \in \mathbb{C}^{N_{\mathrm{t}} \times N_{\mathrm{t}}}$ are unitary. While, with Gaussian signaling, $\mathbf{d}$ would be unitarily invariant and thus $\mathbf{V}_{\mathbf{B}}$ would be an identity matrix, for the signals at hand $\mathbf{V}_{\mathbf{B}}$ plays an important role.

When Gaussian-signal precoding solutions are applied to discrete constellations, the performance suffers because, in the face of major power discrepancies among MIMO subchannels, these solutions insist on beamforming over an extensive range of signal-to-noise ratios (SNRs), well beyond the point where beamforming is appropriate for a discrete constellation. With beamforming, signalling occurs only over the dominant subchannel, which causes a performance loss with discrete signals [3], [20]. By properly designing $\mathbf{U}_{\mathbf{B}}, \Lambda_{\mathbf{B}}$, and $\mathbf{V}_{\mathbf{B}}$, this loss can be eliminated [3], [20]. The matrix $\mathbf{V}_{\mathbf{B}}$ mixes the $N_{\mathrm{t}}$ original signals into $N_{\mathrm{t}}$ beams, then $\Lambda_{\mathrm{B}}$ allocates power to those beams, and finally $\mathbf{U}_{\mathbf{B}}$ aligns them spatially as

\footnotetext{
${ }^{1}$ The channel model in (2) is only intended for Example 1, to explain the basic idea behind the low complexity design; it does not represent the jointly correlated Rician fading model analyzed in Section IV.
} 
they are launched onto the channel. With a proper choice of $\mathbf{V}_{\mathbf{B}}$, in particular, all the $N_{\mathrm{t}}$ signals can be effectively transmitted even if only a single beam is active.

\section{Example 1:}

In a $4 \times 4$ MIMO channel,

$$
\mathrm{y}=\mathrm{HU}_{\mathrm{B}} \Lambda_{\mathrm{B}} \mathrm{V}_{\mathrm{B}} \mathrm{d}+\mathbf{n}
$$

where $\mathbf{H}=\mathbf{U}_{\mathbf{H}} \boldsymbol{\Lambda}_{\mathbf{H}} \mathbf{V}_{\mathbf{H}}$ and $\mathbf{d}=\left[d_{1}, d_{2}, d_{3}, d_{4}\right]^{T}$. Here, $\mathbf{U}_{\mathbf{H}} \in \mathbb{C}^{4 \times 4}$ and $\mathbf{V}_{\mathbf{H}} \in \mathbb{C}^{4 \times 4}$ are unitary matrices, and $\Lambda_{\mathbf{H}} \in \mathbb{C}^{4 \times 4}$ is a diagonal matrix. If $\mathbf{H}$ is known by the transmitter, then, from [3, Prop. 2], the optimal design satisfies $\mathbf{U}_{\mathbf{B}}=\mathbf{V}_{\mathbf{H}}^{H}$ and (4) becomes

$$
\overline{\mathbf{y}}=\left[\begin{array}{ccc}
a_{1} \lambda_{1} & & \\
& \ddots & \\
& & a_{4} \lambda_{4}
\end{array}\right]\left[\begin{array}{ccc}
V_{11} & \ldots & V_{14} \\
\vdots & \ddots & \vdots \\
V_{41} & \ldots & V_{44}
\end{array}\right] \mathbf{d}+\mathbf{n}
$$

where $\overline{\mathbf{y}}=\mathbf{U}_{\mathbf{H}}^{H} \mathbf{y}$ while $a_{i}$ and $\lambda_{i}$ are the diagonal entries of $\boldsymbol{\Lambda}_{\mathbf{H}}$ and $\boldsymbol{\Lambda}_{\mathbf{B}}$, respectively, and $V_{i j}=\left[\mathbf{V}_{\mathbf{B}}\right]_{i j}$.

Suppose that two of the subchannel gains, say $a_{2}$ and $a_{4}$, are very weak. Then, with a Gaussian-signal precoder, the powers allocated to the corresponding subchannels will be very small even at moderate SNRs. Since, with Gaussian signals, $\mathbf{V}_{\mathbf{B}}$ is an identity matrix, $d_{2}$ and $d_{4}$ are essentially muted. With a proper $\mathbf{V}_{\mathbf{B}}$, in contrast, the received signal satisfies

$$
[\overline{\mathbf{y}}]_{i}=a_{i} \lambda_{i} \sum_{j=1}^{4} V_{i j} d_{j} \quad i=1,2,3,4
$$

and now, even if $a_{2} \lambda_{2} \approx 0$ and $a_{4} \lambda_{4} \approx 0, d_{2}$ and $d_{4}$ can still be effectively transmitted along other subchannels.

As indicated by (6), an adequate design for discrete constellations generally mixes all the signals $\left(d_{1}, d_{2}, d_{3}, d_{4}\right)$ and transmits the ensuing beams on different subchannels. This is referred as a complete search design. In fact, for single-user MIMO systems with finite-alphabet inputs, a complete search design can achieve the maximal mutual information and near-maximal mutual information with instantaneous CSI [3] and statistical CSI [20] at the transmitter, respectively. However, the search space grows exponentially with $N_{\mathrm{t}}[3]$. 
TABLE I: Precoder designs for single-user MIMO channel with finite alphabet inputs

\begin{tabular}{|c|c|c|c|}
\hline Paper & CSI at Transmitter & Precoder Structure & Performance \\
\hline C. Xiao et al. [3] & Instantaneous CSI & Complete search & Optimal \\
\hline W. Zeng et al. [20] & $\begin{array}{c}\text { Statistical CSI } \\
\text { Kronecker fading }\end{array}$ & Near-optimal \\
\hline S. K. Mohammed et al. [9] & Instantaneous CSI & Per-group search with fixed $\boldsymbol{\Lambda}_{\mathbf{B}}$ and $\mathbf{V}_{\mathbf{B}}$ & Suboptimal \\
\hline T. Ketseoglou et al. [10] & Instantaneous CSI & Per-group search with optimized $\boldsymbol{\Lambda}_{\mathbf{B}}$ and $\mathbf{V}_{\mathbf{B}}$ & Near-optimal \\
\hline The work in this paper & Statistical CSI & Per-group search with optimized $\boldsymbol{\Lambda}_{\mathbf{B}}$ and $\mathbf{V}_{\mathbf{B}}$ & Near-optimal \\
\hline
\end{tabular}

Intuitively though, if there are two weak subchannels, say $a_{2}$ and $a_{4}$ in Example 1, it is not necessary to mix all the signals. It suffices to mix $d_{2}$ with $d_{1}$, and $d_{4}$ with $d_{3}$, and then transmit the ensuing beams on the strong subchannels $a_{1}$ and $a_{3}$. This corresponds to

$$
\mathbf{V}_{\mathbf{B}}=\left[\begin{array}{cccc}
V_{11} & V_{12} & 0 & 0 \\
V_{21} & V_{22} & 0 & 0 \\
0 & 0 & V_{33} & V_{34} \\
0 & 0 & V_{43} & V_{44}
\end{array}\right]
$$

which, plugged into (5), gives

$$
\begin{array}{rlrl}
{[\overline{\mathbf{y}}]_{i}=a_{i} \lambda_{i} \sum_{j=1}^{2} V_{i j} d_{j}} & i=1,2 \\
{[\overline{\mathbf{y}}]_{i}=a_{i} \lambda_{i} \sum_{j=3}^{4} V_{i j} d_{j}} & i=3,4 .
\end{array}
$$

Observe from (8) and (9) that $\left(d_{1}, d_{2}\right)$ and $\left(d_{3}, d_{4}\right)$ are decoupled. This is referred as a per-group search design. If the entries of $\mathbf{d}$ are QPSK, then the search space is of dimension $2 \times 4^{2 \times 2}=512$ [3]. In contrast, for the complete search in (6), it would be of dimension $4^{2 \times 4}=65536$. As will be seen, this enormous reduction in complexity may incur only a minute loss in performance.

With instantaneous CSI, the idea suggested in Example 1 leads to the PGP technique in [10]. A more general construction that does not require instantaneous CSI at the transmitter is presented next. Table I provides a comparison between the previous work for precoder designs for single-user MIMO with finite-alphabet inputs and the work in this paper. 


\section{Low-CompleXity PRECOder Design}

\section{A. Channel Model}

To avoid modeling artifacts in the design of the precoder, we consider the rather general MIMO channel model

$$
\mathbf{H}=\mathbf{U}_{\mathrm{R}}(\tilde{\mathbf{G}} \odot \mathbf{W}) \mathbf{U}_{\mathrm{T}}^{H}+\overline{\mathbf{H}}
$$

where $\mathbf{U}_{\mathrm{R}}=\left[\mathbf{u}_{\mathrm{R}, 1}, \mathbf{u}_{\mathrm{R}, 2}, \ldots, \mathbf{u}_{\mathrm{R}, N_{\mathrm{r}}}\right] \in \mathbb{C}^{N_{\mathrm{r}} \times N_{\mathrm{r}}}$ and $\mathbf{U}_{\mathrm{T}}=\left[\mathbf{u}_{\mathrm{T}, 1}, \mathbf{u}_{\mathrm{T}, 2}, \ldots, \mathbf{u}_{\mathrm{T}, N_{\mathrm{t}}}\right] \in \mathbb{C}^{N_{\mathrm{t}} \times N_{\mathrm{t}}}$ are deterministic unitary matrices, $\tilde{\mathbf{G}}$ is a deterministic matrix of size $N_{\mathrm{r}} \times N_{\mathrm{t}}$ with real-valued nonnegative entries, $\mathbf{W} \in \mathbb{C}^{N_{\mathrm{r}} \times N_{\mathrm{t}}}$ is a random matrix whose entries are independent and identically distributed (IID) complex Gaussian with zero-mean and unit-variance, and $\overline{\mathbf{H}} \in \mathbb{C}^{N_{\mathrm{r}} \times N_{\mathrm{t}}}$ is a deterministic matrix modeling the Rice component. We further define $\mathbf{G}=\tilde{\mathbf{G}} \odot \tilde{\mathbf{G}}$ such that $[\mathbf{G}]_{n m}$ is the average power coupling between $\mathbf{u}_{\mathrm{R}, n}$ and $\mathbf{u}_{\mathrm{T}, m}$ [22]. The transmit and receive correlation matrices of $\mathbf{H}$ are

$$
\begin{aligned}
& \mathbf{R}_{\mathrm{t}}=E_{\mathbf{H}}\left[(\mathbf{H}-\overline{\mathbf{H}})^{H}(\mathbf{H}-\overline{\mathbf{H}})\right]=\mathbf{U}_{\mathrm{T}} \boldsymbol{\Gamma}_{\mathrm{T}} \mathbf{U}_{\mathrm{T}}^{H} \\
& \mathbf{R}_{\mathrm{r}}=E_{\mathbf{H}}\left[(\mathbf{H}-\overline{\mathbf{H}})(\mathbf{H}-\overline{\mathbf{H}})^{H}\right]=\mathbf{U}_{\mathrm{R}} \boldsymbol{\Gamma}_{\mathrm{R}} \mathbf{U}_{\mathrm{R}}^{H}
\end{aligned}
$$

where $\boldsymbol{\Gamma}_{\mathrm{T}}$ and $\boldsymbol{\Gamma}_{\mathrm{R}}$ are diagonal with $\left[\boldsymbol{\Gamma}_{\mathrm{T}}\right]_{m m}=\sum_{n=1}^{N_{\mathrm{r}}}[\mathbf{G}]_{n m}$, for $m=1,2, \ldots, N_{\mathrm{t}}$, and $\left[\boldsymbol{\Gamma}_{\mathrm{R}}\right]_{n n}=$ $\sum_{m=1}^{N_{\mathrm{t}}}[\mathbf{G}]_{n m}$, for $n=1,2, \ldots, N_{\mathrm{r}}$, respectively.

We note that (10) subsumes most statistical MIMO channel models. For instance, if $\overline{\mathbf{H}}=\mathbf{0}$ and $\mathbf{G}$ is rank-one, the Kronecker model is recovered [23]-[25]. Allowing $\mathrm{G}$ to have arbitrary rank while fixing $\mathrm{U}_{\mathrm{R}}$ and $\mathrm{U}_{\mathrm{T}}$ to be Fourier matrices, we obtain the virtual channel representation for uniform linear arrays (ULA) [26]. If we further relax $\mathrm{U}_{\mathrm{R}}$ and $\mathrm{U}_{\mathrm{T}}$ to be arbitrary unitary matrices, we obtain the Weichselberger's channel model [22]. As far as the Rice component is concerned, and in contrast with works where its structure is restricted [15], [27], in our model it is also arbitrary.

Without loss of generality, we normalize $\mathbf{G}$ and $\overline{\mathbf{H}}$ such that

$$
\begin{aligned}
& \frac{1}{N_{\mathrm{r}} N_{\mathrm{t}}}\|\mathbf{G}\|_{F}=\frac{1}{K} \\
& \frac{1}{N_{\mathrm{r}} N_{\mathrm{t}}}\|\overline{\mathbf{H}}\|_{F}^{2}=\frac{K}{K+1}
\end{aligned}
$$

where $K$ is the Rice factor. For $K \rightarrow \infty$ and $K=0$, (10) reduces to a deterministic channel and a Rayleigh-faded channel, respectively.

In this work, we assume that the receiver knows $\mathbf{H}$ perfectly whereas the transmitter only has statistical knowledge thereof, i.e., the transmitter only knows $\overline{\mathbf{H}}, \mathbf{U}_{\mathrm{R}}, \tilde{\mathbf{G}}$, and $\mathbf{U}_{\mathrm{T}}$. As indicated in [28, Table II], 
the coherence time of the channel statistics exceeds $1 \mathrm{~s}$ in typical residential urban environments2. The Long Term Evolution (LTE) specification defines a subframe as a transmission time interval of $1 \mathrm{~ms}$ [32]. Therefore, once $\overline{\mathbf{H}}, \mathbf{U}_{\mathrm{R}}, \tilde{\mathbf{G}}$, and $\mathbf{U}_{\mathrm{T}}$ are obtained and fed back to the transmitter, they can be used for hundreds of subframes. As a result, the overall feedback overhead for precoder designs that are based on statistical CSI is much smaller than that of precoder designs requiring instantaneous CSI3.

With $\mathbf{H}$ known at the receiver, the ergodic mutual information between $\mathbf{x}$ and $\mathbf{y}$ is given by [34]

$$
I(\mathbf{x} ; \mathbf{y})=E_{\mathbf{H}}\left[E_{\mathbf{x}, \mathbf{y}}\left[\log \frac{p(\mathbf{y} \mid \mathbf{x}, \mathbf{H})}{p(\mathbf{y} \mid \mathbf{H})} \mid \mathbf{H}\right]\right]
$$

where the outer expectation is over $\mathbf{H}$ and the inner expectation is over $p(\mathbf{x}, \mathbf{y} \mid \mathbf{H})$.

\section{B. Mutual Information in the Large-Dimensional Regime}

The ergodic mutual information in (14) requires the expectation with respect to the distribution of $\mathbf{H}$, which can not be obtained in closed form. To overcome this problem, the concept of the deterministic equivalent channel [35] can be exploited to approximate (14) in the large-dimensional regime. Using the deterministic equivalent channel, we can then obtain the counterparts to (8) and (9) for the general setting. To this end, we assume that both $N_{\mathrm{r}}$ and $N_{\mathrm{t}}$ grow large with ratio $c=N_{\mathrm{t}} / N_{\mathrm{r}}$. In the following, we define this deterministic equivalent channel and the parameters used to compute its mutual information.

Let us define the vector channel

$$
\mathbf{z}=\Xi^{1 / 2} \mathbf{X}+\check{\mathbf{n}}
$$

where $\Xi$ is the deterministic equivalent channel matrix used to approximate the exact ergodic mutual information in (14) and $\check{\mathbf{n}} \in \mathbb{C}^{N_{\mathrm{t}} \times 1}$ is a standard complex Gaussian random vector. The minimum meansquare error (MMSE) estimate of $\mathbf{x}$ based on the observation of $\mathbf{z}$ is

$$
\hat{\mathbf{x}}(\mathbf{z})=E[\mathbf{x} \mid \mathbf{z}]
$$

\footnotetext{
${ }^{2}$ Measurements for a single-input single-output (SISO) narrowband system were presented in [28]. In general, the channel coherence time is mainly determined by the velocity of the user and the carrier frequency [29. Eq. (5.40)]. The number of transmit and receive antennas has little impact on the channel coherence time. Moreover, it is proved in [30 Prop. 1] that the channel statistics is independent of the frequency for a wideband system. Therefore, for the coherence time of the channel statistics, there is no obvious difference between a SISO narrowband system and a MIMO wideband system.

${ }^{3}$ For precoder designs requiring instantaneous CSI, the feedback overhead can also be reduced by exploiting vector quantization [33].
} 
where the expectation is over $p(\mathbf{x} \mid \mathbf{z})$. The covariance of the estimation error is the MMSE matrix [3], [8], [36]

$$
\boldsymbol{\Omega}=E\left[(\mathbf{x}-\hat{\mathbf{x}}(\mathbf{z}))(\mathbf{x}-\hat{\mathbf{x}}(\mathbf{z}))^{H}\right]
$$

with expectation over $\mathrm{x}$ and $\mathrm{z}$.

Next, we introduce several useful quantities. Define $\gamma=\left[\gamma_{1}, \gamma_{2}, \ldots, \gamma_{N_{\mathrm{r}}}\right]^{T}, \boldsymbol{\psi}=\left[\psi_{1}, \psi_{2}, \ldots, \psi_{N_{\mathrm{t}}}\right]^{T}$, and

$$
\boldsymbol{\Xi}=\mathbf{T}+\overline{\mathbf{H}}^{H}\left(\mathbf{I}_{N_{\mathrm{r}}}+\mathbf{R}\right)^{-1} \overline{\mathbf{H}} \quad \in \mathbb{C}^{N_{\mathrm{t}} \times N_{\mathrm{t}}}
$$

The equivalent channel matrix $\Xi$ is a function of the auxiliary variables $\{\gamma, \boldsymbol{\psi}, \mathbf{R}, \mathbf{T}\}$, which satisfy the coupled equations

$$
\begin{array}{ll}
\mathbf{T}=\mathbf{U}_{\mathrm{T}}^{H} \operatorname{diag}\left(\mathbf{G}^{T} \boldsymbol{\gamma}\right) \mathbf{U}_{\mathrm{T}} & \in \mathbb{C}^{N_{\mathrm{t}} \times N_{\mathrm{t}}} \\
\mathbf{R}=\mathbf{U}_{\mathrm{R}}^{H} \operatorname{diag}(\mathbf{G} \boldsymbol{\psi}) \mathbf{U}_{\mathrm{R}} & \in \mathbb{C}^{N_{\mathrm{r}} \times N_{\mathrm{r}}}
\end{array}
$$

while the entries of $\gamma$ and $\psi$ are the solutions to the fixed-point equations

$$
\begin{aligned}
& \gamma_{m}=\mathbf{u}_{\mathrm{R}, m}^{H}\left(\mathbf{I}_{N_{\mathrm{r}}}+\mathbf{R}\right)^{-1} \mathbf{u}_{\mathrm{R}, m}-\mathbf{u}_{\mathrm{R}, m}^{H}\left(\mathbf{I}_{N_{\mathrm{r}}}+\mathbf{R}\right)^{-1} \overline{\mathbf{H}} \boldsymbol{\Omega} \overline{\mathbf{H}}^{H}\left(\mathbf{I}_{N_{\mathrm{r}}}+\mathbf{R}\right)^{-1} \mathbf{u}_{\mathrm{R}, m} \\
& \psi_{n}=\mathbf{u}_{\mathrm{T}, n}^{H} \boldsymbol{\Omega} \mathbf{u}_{\mathrm{T}, n} .
\end{aligned}
$$

The equivalent channel matrix $\Xi$ in $(15)$ does not depend on the instantaneous channel realizations, but it is merely an instrument to obtain an asymptotic expression for the ergodic mutual information in (14), which is given as follows.

Proposition 1: In the large-dimensional regime, the mutual information in (14) satisfies

$$
I(\mathbf{x} ; \mathbf{y}) \simeq I_{\text {asy }}(\mathbf{x} ; \mathbf{y})
$$

where

$$
I_{\text {asy }}(\mathbf{x} ; \mathbf{y})=I(\mathbf{x} ; \mathbf{z})+\log \operatorname{det}\left(\mathbf{I}_{N_{\mathrm{r}}}+\mathbf{R}\right)-\boldsymbol{\gamma}^{T} \mathbf{G} \boldsymbol{\psi}
$$

with $I(\mathbf{x} ; \mathbf{z})$ being the mutual information over the equivalent channel in (15). The approximation in (23) sharpens as the matrices become large.

Proof: See Appendix A.

Remark 1: We note that there are three main differences between the asymptotic expression in Proposition 1 and the asymptotic expression in [31]. First, our asymptotic expression and the asymptotic expression 
in [31] apply for the mutual information with finite alphabet inputs and Gaussian inputs, respectively. Therefore, the employed mathematical methods are completely different. The derivation of the asymptotic expressions relies the replica method and the Stieltjes transform for finite alphabet inputs and Gaussian inputs, respectively. Second, our expression applies for correlated fading channels while the expression in [31] only applies for independent fading channels. Third, our expression accounts for the Rician factor.

In the following, we shall take advantage of the asymptotic mutual information expression in Proposition 1 to design the precoder $\mathbf{B}$.

\section{Precoder Structure}

1) Structure of $\mathbf{U}_{\mathbf{B}}$ : Consider the eigenvalue decomposition $\boldsymbol{\Xi}=\mathbf{U}_{\Xi} \boldsymbol{\Lambda}_{\Xi} \mathbf{U}_{\Xi}^{H}$ where $\boldsymbol{\Lambda}_{\Xi} \in \mathbb{C}^{N_{\mathrm{t}} \times N_{\mathrm{t}}}$ is diagonal and $\mathbf{U}_{\Xi} \in \mathbb{C}^{N_{\mathrm{t}} \times N_{\mathrm{t}}}$ is unitary.

\section{Proposition 2:}

The precoder left singular matrix $\mathbf{U}_{\mathbf{B}}$ that maximizes the asymptotic mutual information in (23) equals $\mathrm{U}_{\Xi}$

\section{Proof: See Appendix B.}

This result generalizes what was found in [20] for Kronecker channels, where it is optimal to transmit along the eigendirections of the transmit correlation matrix $\mathrm{U}_{\mathrm{T}}$.

Now, plugging $\mathbf{U}_{\mathbf{B}}=\mathbf{U}_{\Xi}$ into (15) and using [3, (8)], we can rewrite (15) as

$$
\mathbf{z}_{\mathrm{eq}}=\Lambda_{\Xi}^{1 / 2} \mathbf{x}_{\mathrm{eq}}+\check{\mathbf{n}}
$$

where

$$
\begin{aligned}
& \mathbf{z}_{\mathrm{eq}}=\mathbf{U}_{\Xi}^{H} \mathbf{z} \\
& \mathbf{x}_{\mathrm{eq}}=\boldsymbol{\Lambda}_{\mathbf{B}} \mathbf{V}_{\mathbf{B}} \mathbf{d} .
\end{aligned}
$$

Let us divide the transmit signal $\mathbf{d}$ into $S$ streams. Each stream $\mathbf{d}_{\mathrm{s}} \in \mathbb{C}^{N_{\mathrm{s}} \times 1}$ is to be conveyed over $N_{\mathrm{s}}=N_{\mathrm{t}} / S$ diagonal entries of $\boldsymbol{\Lambda}_{\boldsymbol{\Xi}}$. Let the set $\left\{\ell_{1}, \ldots, \ell_{N_{\mathrm{t}}}\right\}$ denote a permutation of $\left\{1, \ldots, N_{\mathrm{t}}\right\}$ and let $\Lambda_{s} \in \mathbb{C}^{N_{\mathrm{s}} \times N_{\mathrm{s}}}$ and $\mathbf{V}_{s} \in \mathbb{C}^{N_{\mathrm{s}} \times N_{\mathrm{s}}}$ denote a diagonal matrix and a unitary matrix, respectively, for $s=1, \ldots, S . \Lambda_{s}$ and $\mathbf{V}_{s}$ will be optimized later. The goal of arranging these $S$ streams as in (8) and (9) prompts the subsequent design steps. 
2) Structure of $\Lambda_{\mathrm{B}}$ : We set

$$
\left[\boldsymbol{\Lambda}_{\mathbf{B}}\right]_{\ell_{j} \ell_{j}}=\left[\boldsymbol{\Lambda}_{s}\right]_{i i}
$$

where $i=1, \ldots, N_{\mathrm{s}}, s=1, \ldots, S$ and $j=(s-1) N_{\mathrm{s}}+i$. With this structure, the $s$ th stream is transmitted along the $\ell_{(s-1) N_{\mathrm{s}}+1}, \ldots, \ell_{(s-1) N_{\mathrm{s}}+N_{\mathrm{s}}}$ diagonal entries of $\boldsymbol{\Lambda}_{\boldsymbol{\Xi}}$.

3) Structure of $\mathrm{V}_{\mathrm{B}}$ : Here we set

$$
\left[\mathbf{V}_{\mathbf{B}}\right]_{\ell_{i} \ell_{j}}= \begin{cases}{\left[\mathbf{V}_{s}\right]_{m n}} & i=(s-1) N_{\mathrm{s}}+m, j=(s-1) N_{\mathrm{s}}+n \\ 0 & \text { otherwise }\end{cases}
$$

for $m=1, \ldots, N_{\mathrm{s}}, n=1, \ldots, N_{\mathrm{s}}, s=1, \ldots, S, i=1, \ldots, N_{\mathrm{t}}$, and $j=1, \ldots, N_{\mathrm{t}}$. With this structure, the $s$ th stream is mapped only to rows $\ell_{(s-1) N_{\mathrm{s}}+1}, \ldots, \ell_{(s-1) N_{\mathrm{s}}+N_{\mathrm{s}}}$ and columns $\ell_{(s-1) N_{\mathrm{s}}+1}, \ldots, \ell_{(s-1) N_{\mathrm{s}}+N_{\mathrm{s}}}$ of $\mathbf{V}_{\mathbf{B}}$. This yields $S$ decoupled groups of streams at the receiver.

The design in (7) is a specific instance of (29) with $\left\{\ell_{1}, \ldots, \ell_{N_{t}}\right\}=\{1,2,3,4\}$ and $S=2$. Recall how $\left(d_{1}, d_{2}\right)$ and $\left(d_{3}, d_{4}\right)$ are indeed decoupled in (8) and (9).

4) Structure of $\mathbf{d}_{s}$ : Finally, we let

$$
\left[\mathbf{d}_{s}\right]_{i}=[\mathbf{d}]_{\ell_{j}}
$$

where $i=1,2, \ldots, N_{\mathrm{s}}, s=1,2, \ldots, S$, and $j=(s-1) N_{\mathrm{s}}+i$.

\section{Precoder Optimization}

Based on (28)-(30), the relationship in (27) becomes

$$
\left[\mathbf{x}_{\mathrm{eq}}\right]_{\ell_{j}}=\left[\boldsymbol{\Lambda}_{s} \mathbf{V}_{s} \mathbf{d}_{s}\right]_{i}
$$

for $i=1, \ldots, N_{\mathrm{s}}, s=1, \ldots, S$, and $j=(s-1) N_{\mathrm{s}}+i$. Recalling that $\boldsymbol{\Lambda}_{\Xi}$ is diagonal, (25) then reduces to

$$
\left[\mathbf{z}_{\mathrm{eq}}\right]_{\ell_{j}}=\left[\boldsymbol{\Lambda}_{\mathbf{\Xi}}\right]_{\ell_{j} \ell_{j}}^{1 / 2}\left[\mathbf{x}_{\mathrm{eq}}\right]_{\ell_{j}}+\left[\mathbf{n}_{s}\right]_{i}
$$

where $\left[\mathbf{n}_{s}\right]_{i}=[\check{\mathbf{n}}]_{\ell_{j}}$.

Equations (31) and (32) indicate that each independent data stream $\mathbf{d}_{s}$ is transmitted along its own $N_{\mathrm{s}}$ separate subchannels without interfering with other streams. Furthermore, the MMSE matrix then equals

$$
[\boldsymbol{\Omega}]_{\ell_{i} \ell_{j}}= \begin{cases}{\left[\boldsymbol{\Omega}_{s}\right]_{m n}} & i=(s-1) N_{\mathrm{s}}+m, j=(s-1) N_{\mathrm{s}}+n \\ 0 & \text { otherwise }\end{cases}
$$


where

$$
\boldsymbol{\Omega}_{s}=\boldsymbol{\Lambda}_{s} \mathbf{V}_{s} E\left[\left(\mathbf{d}_{s}-\hat{\mathbf{d}}_{s}\right)\left(\mathbf{d}_{s}-\hat{\mathbf{d}}_{s}\right)^{H}\right] \mathbf{V}_{s}^{H} \boldsymbol{\Lambda}_{s}^{H}
$$

with

$$
\hat{\mathbf{d}}_{s}=E\left[\mathbf{d}_{s} \mid \mathbf{z}_{s}\right]
$$

and $\left[\mathbf{z}_{s}\right]_{i}=\left[\mathbf{z}_{\mathrm{eq}}\right]_{\ell_{j}}$, and further defining diagonal matrices $\boldsymbol{\Xi}_{s}$, for $s=1, \ldots, S$, with entries $\left[\boldsymbol{\Xi}_{s}\right]_{i i}=$ $\left[\boldsymbol{\Lambda}_{\boldsymbol{\Xi}}\right]_{\ell_{j} \ell_{j}}$

The main term within (24) can now be expressed as

$$
I(\mathbf{x} ; \mathbf{z})=\sum_{s=1}^{S} I\left(\mathbf{d}_{s} ; \mathbf{z}_{s}\right)
$$

based on which the gradients of $I_{\text {asy }}(\mathbf{x} ; \mathbf{y})$ with respect to $\Lambda_{s}^{2}$ and $\mathbf{V}_{s}$ are given by [36, (22)],

$$
\begin{aligned}
& \nabla_{\boldsymbol{\Lambda}_{s}^{2}} I_{\mathrm{asy}}(\mathbf{x} ; \mathbf{y})=\operatorname{diag}\left(\mathbf{V}_{s}^{H} \mathbf{E}_{s} \mathbf{V}_{s} \boldsymbol{\Xi}_{s}\right) \\
& \nabla_{\mathbf{V}_{s}} I_{\text {asy }}(\mathbf{x} ; \mathbf{y})=\boldsymbol{\Xi}_{s} \boldsymbol{\Lambda}_{s}^{2} \mathbf{V}_{s} \mathbf{E}_{s}
\end{aligned}
$$

where

$$
\mathbf{E}_{s}=E\left[\left(\mathbf{d}_{s}-\hat{\mathbf{d}}_{s}\right)\left(\mathbf{d}_{s}-\hat{\mathbf{d}}_{s}\right)^{H}\right]
$$

Based on Propositions 1 and 2, on (36), and on the relationship between $\Lambda_{1}, \ldots, \boldsymbol{\Lambda}_{S}$ and $\boldsymbol{\Lambda}_{\mathbf{B}}$ in (28) as well as the relationship between $\mathbf{V}_{1}, \ldots, \mathbf{V}_{S}$ and $\mathbf{V}_{\mathbf{B}}$ in (29), we propose Algorithm 1 to optimize $\mathbf{B}$. In Steps 3 and 5 of this algorithm, $\Lambda_{s}^{(n)}$ and $\mathbf{V}_{s}^{(n)}$ are updated along the gradient descent direction, with the backtracking line search method used to determine the step size. In Step $4, \Lambda_{s}^{(n)}$ is normalized to satisfy the power constraint. In Step 6, $\boldsymbol{\Xi}, \mathbf{R}, \gamma$, and $\psi$ are updated for the new precoder based on (18)-(22), (33). In Step 7, if $n$ is less than some maximum number of iterations and $I^{(n+1)}(\mathbf{x} ; \mathbf{y})-I^{(n)}(\mathbf{x} ; \mathbf{y})$ is above some threshold, the iterations continue; otherwise, the algorithm is stopped. In Step 8, we compute the optimal $\mathrm{U}_{\mathbf{B}}$ based on Proposition 2 .

With statistical CSI, the expectation of the mutual information in (14) can be evaluated efficiently by applying [20, Prop. 2]. Likewise, operations such as matrix products and the fixed-point equations are polynomial functions of the numbers of antennas, and thus can also be performed efficiently. What dominates the computational cost is expecting the mutual information and the MMSE matrix over $\mathbf{d}_{m}$, as the complexity of these expectations is exponential in $N_{\mathrm{t}}[20$, (14) and (47)]. Therefore, it suffices to

Algorithm 1: Maximization of $I(\mathbf{x} ; \mathbf{y})$ with respect to $\mathbf{B}$. 
TABLE II: Number of additions required to calculate the mutual information and the MMSE matrix for QPSK and $N_{\mathrm{s}}=2$.

\begin{tabular}{|c|c|c|c|c|}
\hline$N_{\mathrm{t}}$ & 4 & 8 & 16 & 32 \\
\hline $\begin{array}{c}\text { Complete-search } \\
\text { design in [20] }\end{array}$ & 65536 & $4.29 \times 10^{9}$ & $1.84 \times 10^{19}$ & $3.4 \times 10^{38}$ \\
\hline Algorithm 1 & 512 & 1024 & 2048 & 4096 \\
\hline
\end{tabular}

1) Initialize $\Lambda_{s}^{(0)}, \mathbf{V}_{s}^{(0)}$ for $s=1, \ldots, S$. Fix a maximum number of iterations, $N_{\text {iter }}$, and a threshold $\varepsilon$.

2) Initialize $\boldsymbol{\Xi}, \mathbf{R}, \boldsymbol{\gamma}$, and $\psi$ based on (18)-(22), with $\Omega$ based on (33). Then, initialize $I^{(1)}(\mathbf{x} ; \mathbf{y})$ based on (23) with $I(\mathbf{x} ; \mathbf{z})$ as per (36). Set counter to $n=1$.

3) Update $\Lambda_{s}^{(n)}$ for $s=1, \ldots, S$ along the gradient descent direction given by (37).

4) Normalize $\sum_{s=1}^{S}\left[\boldsymbol{\Lambda}_{s}^{(n)}\right]^{2}=P$.

5) Update $\mathbf{V}_{s}^{(n)}$ for $s=1, \ldots, S$ along the gradient descent direction in (38).

6) Update $\Xi, \mathbf{R}, \gamma$, and $\psi$ based on (18)-(22), (33).

7) Compute $I^{(n+1)}(\mathbf{x} ; \mathbf{y})$ based on (23) and (36). If $I^{(n+1)}(\mathbf{x} ; \mathbf{y})-I^{(n)}(\mathbf{x} ; \mathbf{y})>\varepsilon$ and $n \leq N_{\text {iter }}^{\max }$, set $n=n+1$ and repeat Steps 3-7.

8) Compute $U_{B}$ from the eigenvalue decomposition of the final $\Xi$.

9) Compute $\Lambda_{B}$ and $V_{B}$ based on (28) and (29). Set $B=U_{B} \Lambda_{B} V_{B}$.

compare the computational complexity of these latter operations. When $N_{\mathrm{t}}$ increases, such complexity for the complete-search design in [20] scales with $M^{2 N_{\mathrm{t}}}$. In contrast, for Algorithm 1] it scales with $S M^{2 N_{\mathrm{s}}}$.

To illustrate how enormous the savings can be, consider an example where $N_{\mathrm{s}}=2$ and the signals are QPSK. The numbers of additions required by the complete-search design and by Algorithm 1 are contrasted in Table $\llbracket$ for different values of $N_{\mathrm{t}}$.

Remark 2: Through $S$ and $N_{\mathrm{s}}$, Algorithm 1 offers a tradeoff between performance and complexity. At one end, for $S=1$ and $N_{\mathrm{s}}=N_{\mathrm{t}}$, Algorithm 1 searches the entire space while, at the other end, for $S=N_{\mathrm{t}}$ and $N_{\mathrm{s}}=1$, it merely allocates power among the $N_{\mathrm{t}}$ parallel subchannels. Varying $N_{\mathrm{s}}$ from 1 to $N_{\mathrm{t}}$ bridges the gap between separate and fully joint transmission of the $N_{\mathrm{t}}$ original signals.

Remark 3: An adequate choice of $\ell_{1}, \ldots, \ell_{N_{\mathrm{t}}}$ is important for Algorithm 1 to perform satisfactorily. The $N_{\mathrm{s}} / 2$ largest diagonal entries of $\left[\boldsymbol{\Xi}_{\text {eq }}\right]$ should be paired with the $N_{\mathrm{s}} / 2$ smallest diagonal entries. Then, the 
$N_{\mathrm{s}} / 2$ next largest diagonal entries of $\left[\boldsymbol{\Xi}_{\text {eq }}\right]$ should be paired with the $N_{\mathrm{s}} / 2$ next smallest ones, and so on.

Remark 4: Since $\Lambda_{s}^{(n)}$ and $\mathbf{V}_{s}^{(n)}$ are searched along gradient descent directions, in Step 7 the mutual information $I^{(n)}(\mathbf{x} ; \mathbf{y})$ is nondecreasing. Algorithm 1 thus generates sequences that are nondecreasing and upper-bounded, hence it is convergent. However, due to the nonconvexity of $I^{(n)}(\mathbf{x} ; \mathbf{y})$ in $\Lambda_{s}^{(n)}$ and $\mathbf{V}_{s}^{(n)}$, Algorithm 1 may only find local optima. As a result, the algorithm is run several times with different initializations of $\Lambda_{s}^{(n)}$ and $\mathbf{V}_{s}^{(n)}$ and the precoder that provides the highest mutual information is retained [37]-[39].

In the following, we provide an example to better illustrate the proposed precoder design based on statistical CSI $\overline{\mathbf{h}}, \mathbf{U}_{\mathrm{R}}, \tilde{\mathbf{G}}$, and $\mathbf{U}_{\mathrm{T}}$.

Example 2: Consider a $1 \times 4$ deterministic channel $\mathbf{h}_{\mathrm{d}}$ with $\operatorname{SVD} \mathbf{h}_{\mathrm{d}}=[a, 0,0,0] \mathbf{U}_{\mathbf{h}}^{H}$ and $a=\left\|\mathbf{h}_{\mathrm{d}}\right\|$. The corresponding received signal is

$$
y=[a, 0,0,0] \mathbf{U}_{\mathbf{h}}^{H} \mathbf{U}_{\mathbf{B}} \boldsymbol{\Lambda}_{\mathbf{B}} \mathbf{V}_{\mathbf{B}} \mathbf{d}+n .
$$

Setting $\mathbf{U}_{\mathbf{B}}=\mathbf{U}_{\mathbf{h}}$ as in [9], [10], we obtain

$$
y=[a, 0,0,0] \mathbf{\Lambda}_{\mathbf{B}} \mathbf{V}_{\mathbf{B}} \mathbf{d}+n .
$$

If the precoder were to mix only signals pairs, i.e., $d_{1}$ with $d_{2}$ and $d_{3}$ with $d_{4}$, then

$$
\mathbf{V}_{\mathbf{B}}=\left[\begin{array}{cccc}
V_{11} & V_{12} & 0 & 0 \\
V_{21} & V_{22} & 0 & 0 \\
0 & 0 & V_{33} & V_{34} \\
0 & 0 & V_{43} & V_{44}
\end{array}\right]
$$

from which

$$
y=a \lambda_{1} V_{11} d_{1}+a \lambda_{1} V_{12} d_{2}+n
$$

which does not contain $d_{3}$ and $d_{4}$. If the entries of $\mathbf{d}$ were BPSK-distributed, the spectral efficiency of (43) could not exceed $2 \mathrm{~b} / \mathrm{s} / \mathrm{Hz}$. However, a $1 \times 4$ channel with BPSK inputs can attain $4 \mathrm{~b} / \mathrm{s} / \mathrm{Hz}$ and thus the precoding is incurring a significant loss.

Things are better for fading $\mathbf{h}$, where the low-complexity precoder relies on $\overline{\mathbf{h}}, \mathbf{U}_{\mathrm{R}}, \tilde{\mathbf{G}}$, and $\mathbf{U}_{\mathrm{T}}$, as then $\mathrm{U}_{\mathrm{B}}=\mathrm{U}_{\Xi}$ which in general does not coincide with $\mathrm{U}_{\mathbf{h}}$; this ensures that all signals reach the receiver. To gauge the difference, we randomly generate a $1 \times 4$ fading channel $\mathbf{h}$ based on (10), wherein $K=1$, 
$\mathbf{U}_{\mathrm{R}}=\mathbf{I}_{N_{\mathrm{r}}}$, and $\mathbf{U}_{\mathrm{T}}$ is a Fourier matrix. Then, we implement Algorithm 1 with $N_{\mathrm{s}}=2$. The spectral efficiency at $\mathrm{SNR}=10 \mathrm{~dB}$ is $2.38 \mathrm{~b} / \mathrm{s} / \mathrm{Hz}$, which exceeds $2 \mathrm{~b} / \mathrm{s} / \mathrm{Hz}$. The corresponding $\Lambda_{\Xi}^{1 / 2}$ is

$$
\Lambda_{\Xi}^{1 / 2}=\left[\begin{array}{cccc}
0.80 & 0 & 0 & 0 \\
0 & 0.14 & 0 & 0 \\
0 & 0 & 0.28 & 0 \\
0 & 0 & 0 & 0.24
\end{array}\right]
$$

which indicates that the equivalent channel matrix $\Xi^{1 / 2}$ in $(15)$ is full-rank. For $N_{\mathrm{s}}=4$, i.e., with full complexity, the spectral efficiency of Algorithm 1 is $2.40 \mathrm{~b} / \mathrm{s} / \mathrm{Hz}$, indicating that the low-complexity precoder with $N_{\mathrm{s}}=2$ is close to optimal.

\section{E. Some Special Cases}

1) Kronecker Channel Model: In the Kronecker model, $\overline{\mathbf{H}}=\mathbf{0}$ and $\mathbf{G}$ is a rank-one matrix of the form

$$
\mathrm{G}=\boldsymbol{\lambda}_{\mathrm{r}} \boldsymbol{\lambda}_{\mathrm{t}}^{T}
$$

where $\boldsymbol{\lambda}_{\mathrm{r}}=\left[\begin{array}{llll}\lambda_{\mathrm{r}, 1} & \lambda_{\mathrm{r}, 2} \ldots \lambda_{\mathrm{r}, N_{\mathrm{r}}}\end{array}\right]^{T} \in \mathbb{R}^{N_{\mathrm{r}}}$ while $\boldsymbol{\lambda}_{\mathrm{t}}=\left[\begin{array}{lll}\lambda_{\mathrm{t}, 1} & \lambda_{\mathrm{t}, 2} \ldots \lambda_{\mathrm{t}, N_{\mathrm{t}}}\end{array}\right]^{T} \in \mathbb{R}^{N_{\mathrm{t}}}$. In this case, (10) can be equivalently written as

$$
\mathbf{H}=\mathbf{A}_{\mathrm{R}}^{1 / 2} \mathbf{W} \mathbf{A}_{\mathrm{T}}^{1 / 2}
$$

where $\mathbf{A}_{\mathrm{R}}=\mathbf{U}_{\mathrm{R}} \operatorname{diag}\left(\boldsymbol{\lambda}_{\mathrm{r}}\right) \mathbf{U}_{\mathrm{R}}^{H}$ and $\mathbf{A}_{\mathrm{T}}=\mathbf{U}_{\mathrm{T}} \operatorname{diag}\left(\boldsymbol{\lambda}_{\mathrm{t}}\right) \mathbf{U}_{\mathrm{T}}^{H}$. Then, (18) and (19) reduce to

$$
\boldsymbol{\Xi}=\mathbf{T}=\gamma^{\circ} \mathbf{A}_{\mathrm{T}}
$$

and

$$
\mathbf{R}=\psi^{\circ} \mathbf{A}_{\mathrm{R}}
$$

where $\gamma^{\circ}=\boldsymbol{\lambda}_{\mathrm{r}}^{T} \gamma$ and $\psi^{\circ}=\boldsymbol{\lambda}_{\mathrm{t}}^{T} \boldsymbol{\psi}$. Thus, from (21),

$$
\begin{aligned}
& \gamma^{\circ}=\operatorname{tr}\left(\left(\mathbf{I}_{N_{\mathrm{r}}}+\mathbf{R}\right)^{-1} \mathbf{A}_{\mathrm{r}_{i_{k}}}\right) \\
& \psi^{\circ}=\operatorname{tr}\left(\boldsymbol{\Omega} \mathbf{A}_{\mathrm{T}}\right) .
\end{aligned}
$$

From (47), the optimal left singular matrix $\mathbf{U}_{\mathbf{B}}$ of the precoder $\mathbf{B}$ for this channel model equals $\mathbf{U}_{\mathrm{T}}$. Hence, the equivalent channel matrix between $\mathbf{x}_{\mathrm{eq}}$ and $\mathbf{z}_{\mathrm{eq}}$ in (25) simplifies to $\sqrt{\gamma^{\circ}} \operatorname{diag}\left(\boldsymbol{\lambda}_{\mathrm{t}}\right)^{1 / 2}$. Also, (49) and (50) indicate that instead of computing $N_{\mathrm{t}}+N_{\mathrm{r}}$ parameters in fix-point equation (21), we need only compute $\gamma^{\circ}$ and $\psi^{\circ}$ in Algorithm 1. Furthermore, the receiver needs to feed back only $\mathbf{A}_{\mathrm{T}}$ and $\mathbf{A}_{\mathrm{R}}$ to the transmitter for precoder design. 
2) Deterministic Channel: For $K \rightarrow \infty$, the random portion of the channel vanishes and (23) becomes

$$
I_{\text {asy }}(\mathbf{x} ; \mathbf{y})=I(\mathbf{x} ; \overline{\mathbf{H}} \mathbf{x}+\check{\mathbf{n}})
$$

which is exact regardless of the dimensionality. In this case, the receiver only needs to feed back $\overline{\mathbf{H}}$ to the transmitter for precoder design.

3) Massive MIMO: In some cases, by exploiting the spatial characteristics of physical channels, the structure of $\overline{\mathbf{H}}$ can acquire a particular relationship with respect to $\mathrm{U}_{\mathrm{T}}$ and $\mathrm{U}_{\mathrm{R}}$. Then, Algorithm 1 can be simplified.

Assume there are $L+1$ independent paths between the transmitter and the receiver, where the 0th path is the LOS path. Let $c_{l}, \phi_{l, d}$, and $\theta_{l, a}$ denote the attenuation, the angle of departure, and the angle of arrival for the $l$ th path. Then, the $N_{\mathrm{r}} \times N_{\mathrm{t}}$ MIMO channel can be modeled as [40, Sec. 7.3.2]

$$
\mathbf{H}=c_{0} e^{-j 2 \pi d_{0} / \lambda_{c}} \mathbf{u}_{\mathrm{r}}\left(\theta_{0, a}\right) \mathbf{u}_{\mathrm{t}}^{H}\left(\phi_{0, d}\right)+\sum_{l=1}^{L} c_{l} e^{-j 2 \pi d_{l} / \lambda_{\mathrm{c}}} \mathbf{u}_{\mathrm{r}}\left(\theta_{l, a}\right) \mathbf{u}_{\mathrm{t}}^{H}\left(\phi_{l, d}\right)
$$

where $d_{l}$ denotes the distance between transmit antenna 1 and receive antenna 1 along path $l$ and $\lambda_{\mathrm{c}}$ denotes the wavelength; $\mathbf{u}_{\mathrm{t}}(\phi) \in \mathbb{C}^{N_{\mathrm{t}} \times 1}$ and $\mathbf{u}_{\mathrm{r}}(\theta) \in \mathbb{C}^{N_{\mathrm{r}} \times 1}$ are the unit-norm transmit and receive array response vectors.

In massive MIMO [41], the array response vectors become asymptotically orthogonal [42], [43], i.e.,

$$
\lim _{N_{\mathrm{t}} \rightarrow \infty} \mathbf{u}_{\mathrm{t}}^{H}\left(\phi_{p}\right) \mathbf{u}_{\mathrm{t}}\left(\phi_{l}\right)=\delta(p-l)
$$

where $\delta(p-l)$ denotes the Dirac delta pulse. Under this condition, the channel matrix in (52) can be rewritten as

$$
\begin{aligned}
\mathbf{H} & =\sum_{n=1}^{N_{\mathrm{r}}} \sum_{m=1}^{N_{\mathrm{t}}}[\tilde{\mathbf{H}}+\hat{\mathbf{H}}]_{n m} \mathbf{u}_{\mathrm{r}}\left(\theta_{n}\right) \mathbf{u}_{\mathrm{t}}^{H}\left(\phi_{m}\right) \\
& =\mathbf{U}_{\mathrm{R}}(\tilde{\mathbf{H}}+\hat{\mathbf{H}}) \mathbf{U}_{\mathrm{T}}^{H}
\end{aligned}
$$

where $\mathbf{U}_{\mathrm{T}}=\left[\mathbf{u}_{\mathrm{t}}\left(\phi_{1}\right), \mathbf{u}_{\mathrm{t}}\left(\phi_{2}\right), \ldots, \mathbf{u}_{\mathrm{t}}\left(\phi_{N_{\mathrm{t}}}\right)\right]$ and $\mathbf{U}_{\mathrm{R}}=\left[\mathbf{u}_{\mathrm{r}}\left(\theta_{1}\right), \mathbf{u}_{\mathrm{r}}\left(\theta_{2}\right), \ldots, \mathbf{u}_{\mathrm{r}}\left(\theta_{N_{\mathrm{r}}}\right)\right]$ are unitary. Then, the entries of $\widetilde{\mathbf{H}}$ and $\hat{\mathbf{H}}$ satisfy [26]

$$
\begin{aligned}
& {[\tilde{\mathbf{H}}]_{n m} \simeq \sum_{l \in F_{r, n} \cap F_{t, m}} c_{l} e^{-j 2 \pi d_{l} / \lambda_{\mathrm{c}}}} \\
& {[\hat{\mathbf{H}}]_{n m} \simeq \begin{cases}c_{0} e^{-j 2 \pi d_{0} / \lambda_{\mathrm{c}}} & T(n, m)=1 \\
0 & \text { otherwise. }\end{cases} }
\end{aligned}
$$


where $F_{r, n}$ and $F_{t, m}$ denote the subsets of paths whose angles are closest to $\theta_{n}$ and $\phi_{m}$, respectively. In turn, $T(n, m)=1$ if the angles of the LOS path are closest to $\theta_{n}$ and $\phi_{m}$ simultaneously; for other $n$ and $m$, conversely, $T(n, m)=0$. It should be noted that the approximations in (56) and (57) become exact when the dimension of the antenna tends to infinity [44].

Stacking the columns of $\mathbf{H}$ into a vector, we obtain

$$
\operatorname{vec}(\mathbf{H})=\sum_{n=1}^{N_{\mathrm{r}}} \sum_{m=1}^{N_{\mathrm{t}}}\left([\tilde{\mathbf{H}}]_{n m}+[\hat{\mathbf{H}}]_{n m}\right)\left(\mathbf{u}_{\mathrm{t}}^{*}\left(\phi_{m}\right) \otimes \mathbf{u}_{\mathrm{r}}\left(\theta_{n}\right)\right)
$$

from which the correlations within $\mathbf{H}$ are completely characterized as

$$
\begin{gathered}
E\left[\operatorname{vec}(\mathbf{H}) \operatorname{vec}(\mathbf{H})^{H}\right]=\sum_{n=1}^{N_{\mathrm{r}}} \sum_{m=1}^{N_{\mathrm{t}}} E\left[[\tilde{\mathbf{H}}]_{n m}\left[\tilde{\mathbf{H}}^{H}\right]_{n m}\right]\left(\mathbf{u}_{\mathrm{t}}^{*}\left(\phi_{m}\right) \otimes \mathbf{u}_{\mathrm{r}}\left(\theta_{n}\right)\right)\left(\mathbf{u}_{\mathrm{t}}^{*}\left(\phi_{m}\right) \otimes \mathbf{u}_{\mathrm{r}}\left(\theta_{n}\right)\right)^{H} \\
+\sum_{n=1}^{N_{\mathrm{r}}} \sum_{m=1}^{N_{\mathrm{t}}}\left([\hat{\mathbf{H}}]_{n m}\left[\hat{\mathbf{H}}^{H}\right]_{n m}\right)\left(\mathbf{u}_{\mathrm{t}}^{*}\left(\phi_{m}\right) \otimes \mathbf{u}_{\mathrm{r}}\left(\theta_{n}\right)\right)\left(\mathbf{u}_{\mathrm{t}}^{*}\left(\phi_{m}\right) \otimes \mathbf{u}_{\mathrm{r}}\left(\theta_{n}\right)\right)^{H} .
\end{gathered}
$$

The first term on the right-side of (59) equals the correlation matrix of the first term on the right-side of (10). Thus,

$$
\mathbf{G}=E\left[\tilde{\mathbf{H}} \odot \tilde{\mathbf{H}}^{*}\right]
$$

Comparing (10) and (55), we have that

$$
\begin{aligned}
& \tilde{\mathbf{H}}=\tilde{\mathbf{G}} \odot \mathbf{W} \\
& \overline{\mathbf{H}}=\mathbf{U}_{\mathrm{R}} \hat{\mathbf{H}} \mathbf{U}_{\mathrm{T}}^{H}
\end{aligned}
$$

which relate the massive MIMO channel with the model used in our analysis. The sum of fading paths in (56) can be modeled as a Gaussian random variable with variance $[\mathbf{G}]_{i j}$ while the LOS path can be modeled as a rank-one matrix having the same transmit and receive eigendirections as the fading paths, i.e., $\mathrm{U}_{\mathrm{T}}$ and $\mathrm{U}_{\mathrm{R}}$.

From Proposition 2, the optimal $\mathbf{U}_{\mathrm{B}}$ equals $\mathbf{U}_{\mathrm{T}}$. Plugging such matrix into (2), using [20, (5)] and recalling (61), 62), we can re-write (2) as

$$
\mathbf{y}_{\text {phy }}=\mathbf{H}_{\text {phy }} \mathbf{x}_{\text {phy }}+\mathbf{n}
$$

where

$$
\begin{aligned}
\mathbf{x}_{\text {phy }} & =\mathbf{\Lambda}_{\mathbf{B}} \mathbf{V}_{\mathbf{B}} \mathbf{d} \\
\mathbf{H}_{\text {phy }} & =(\tilde{\mathbf{G}} \odot \mathbf{W})+\hat{\mathbf{H}} .
\end{aligned}
$$


With that, (18) becomes

$$
\boldsymbol{\Xi}_{\text {phy }}=\mathbf{T}_{\text {phy }}+\hat{\mathbf{H}}^{H}\left(\mathbf{I}_{N_{\mathrm{r}}}+\mathbf{R}_{\text {phy }}\right)^{-1} \hat{\mathbf{H}}
$$

where

$$
\begin{gathered}
\mathbf{T}_{\text {phy }}=\operatorname{diag}\left(\mathbf{G}^{T} \boldsymbol{\gamma}_{\text {phy }}\right) \\
\mathbf{R}_{\text {phy }}=\operatorname{diag}\left(\mathbf{G} \boldsymbol{\psi}_{\text {phy }}\right) .
\end{gathered}
$$

The entries of $\gamma_{\text {phy }}$ and $\psi_{\text {phy }}$ are the solution to the fixed point-equations

$$
\begin{aligned}
{\left[\boldsymbol{\gamma}_{\mathrm{phy}}\right]_{m} } & =\left[\left(\mathbf{I}_{N_{\mathrm{r}}}+\mathbf{R}_{\text {phy }}\right)^{-1}\left(\mathbf{I}_{N_{\mathrm{r}}}-\hat{\mathbf{H}} \boldsymbol{\Omega}_{\mathrm{phy}} \hat{\mathbf{H}}^{H}\left(\mathbf{I}_{N_{\mathrm{r}}}+\mathbf{R}_{\text {phy }}\right)^{-1}\right)\right]_{m m} \\
{\left[\boldsymbol{\psi}_{\text {phy }}\right]_{n} } & =\left[\boldsymbol{\Omega}_{\text {phy }}\right]_{n n}
\end{aligned}
$$

where

$$
\begin{aligned}
\Omega_{\text {phy }} & =E\left[\left(\mathbf{x}_{\text {phy }}-\hat{\mathbf{x}}_{\text {phy }}\right)\left(\mathbf{x}_{\text {phy }}-\hat{\mathbf{x}}_{\text {phy }}\right)^{H}\right] \\
\hat{\mathbf{x}}_{\text {phy }} & =E\left[\mathbf{x}_{\text {phy }} \mid \mathbf{z}\right] .
\end{aligned}
$$

In massive MIMO, altogether, Algorithm 1 can be simplified in two ways. First, Step 8 is rendered unnecessary since $\mathbf{U}_{\mathrm{B}}=\mathrm{U}_{\mathrm{T}}$. Second, in Steps 2 and 6 the fixed-point equations (66) - (69) involve only diagonal matrices, with the ensuing computational simplification. Furthermore, the receiver needs to feed back the non-zero elements of $\hat{\mathbf{H}}$ in $(57), \tilde{G}, \mathbf{U}_{R}$, and $\mathrm{U}_{\mathrm{T}}$ to the transmitter for precoder design.

\section{Performance Evaluation}

First, let us evaluate the complexity of Algorithm 1 for different values of $N_{\mathrm{s}}$. Tables II-IV provide the number of additions required to calculate the mutual information and the MMSE matrix per iteration of Algorithm 1 for various numbers of antennas and different signal constellations. As anticipated, for $N_{\mathrm{s}}=N_{\mathrm{t}}$, the computational complexity grows exponentially with $N_{\mathrm{t}}$ and quickly becomes unmanageable.

Fig. 1 compares the spectral efficiency vs. Rice factor $K$ for the channel in (10) with $N_{\mathrm{t}}=N_{\mathrm{r}}=4$, $\mathrm{SNR}=15 \mathrm{~dB}$, and QPSK. $\mathrm{U}_{\mathrm{R}}, \mathrm{U}_{\mathrm{T}}$, and $\tilde{\mathrm{G}}$ in (10) are generated randomly. The Rice component in (10) is generated based on the physical channel model in (52). As illustrated in Fig. 1, even for a small number of antennas, the spectral efficiency of the proposed low complexity design with $N_{\mathrm{s}}=2$ is close to the spectral efficiency of the complete search design with $N_{\mathrm{s}}=4$ for a large range of Rician factors 
TABLE III: Number of additions required to calculate the mutual information and the MMSE matrix with BPSK

\begin{tabular}{|c|c|c|c|}
\hline$N_{\mathrm{t}}$ & $N_{\mathrm{s}}=2$ & $N_{\mathrm{s}}=4$ & $N_{\mathrm{s}}=N_{\mathrm{t}}$ \\
\hline 4 & 32 & 256 & 256 \\
\hline 8 & 64 & 512 & 65536 \\
\hline 16 & 128 & 1024 & $4.2950 \mathrm{e}+009$ \\
\hline 32 & 256 & 2048 & $1.8447 \mathrm{e}+019$ \\
\hline
\end{tabular}

TABLE IV: Number of additions required to calculate the mutual information and the MMSE matrix with QPSK

\begin{tabular}{|c|c|c|c|}
\hline$N_{\mathrm{t}}$ & $N_{\mathrm{s}}=2$ & $N_{\mathrm{s}}=4$ & $N_{\mathrm{s}}=N_{\mathrm{t}}$ \\
\hline 4 & 512 & 65536 & 65536 \\
\hline 8 & 1024 & 131072 & $4.2950 \mathrm{e}+009$ \\
\hline 16 & 2048 & 262144 & $1.8447 \mathrm{e}+019$ \\
\hline 32 & 4096 & 524288 & $3.4028 \mathrm{e}+038$ \\
\hline
\end{tabular}

TABLE V: Number of additions required to calculate the mutual information and the MMSE matrix with 16-QAM.

\begin{tabular}{|c|c|c|}
\hline$N_{\mathrm{t}}$ & $N_{\mathrm{s}}=2$ & $N_{\mathrm{s}}=N_{\mathrm{t}}$ \\
\hline 4 & 512 & $4.2950 \mathrm{e}+009$ \\
\hline 8 & 1024 & $1.8447 \mathrm{e}+019$ \\
\hline 16 & 2048 & $3.4028 \mathrm{e}+038$ \\
\hline 32 & 4096 & $1.1579 \mathrm{e}+077$ \\
\hline
\end{tabular}

$K$. Also, the approximated spectral efficiency in (23), denoted by "Asymptotic" in Fig. 1, is close to the exact spectral efficiency in (14). The exact expression in (14) is computed via a Monte Carlo average over the channel matrix $\mathbf{H}$.

Next, we examine Algorithm 1 for practical channels. We adopt the 3GPP SCM [21] for the urban scenario, half-wavelengh antenna spacing at transmitter and receiver, respectively, a velocity of $36 \mathrm{~km} / \mathrm{h}$, and 6 paths. We obtain $\overline{\mathbf{H}}, \mathbf{U}_{\mathrm{R}}, \tilde{\mathrm{G}}$, and $\mathrm{U}_{\mathrm{T}}$ based on a large number of these realizations for the SCM model, and use them for precoder design.

Fig. 2 depicts the spectral efficiency for the 3GPP SCM for different precoder designs with $N_{\mathrm{t}}=N_{\mathrm{r}}=4$ and QPSK. A Gauss-Seidel algorithm with stochastic programming is employed to obtain the capacity- 

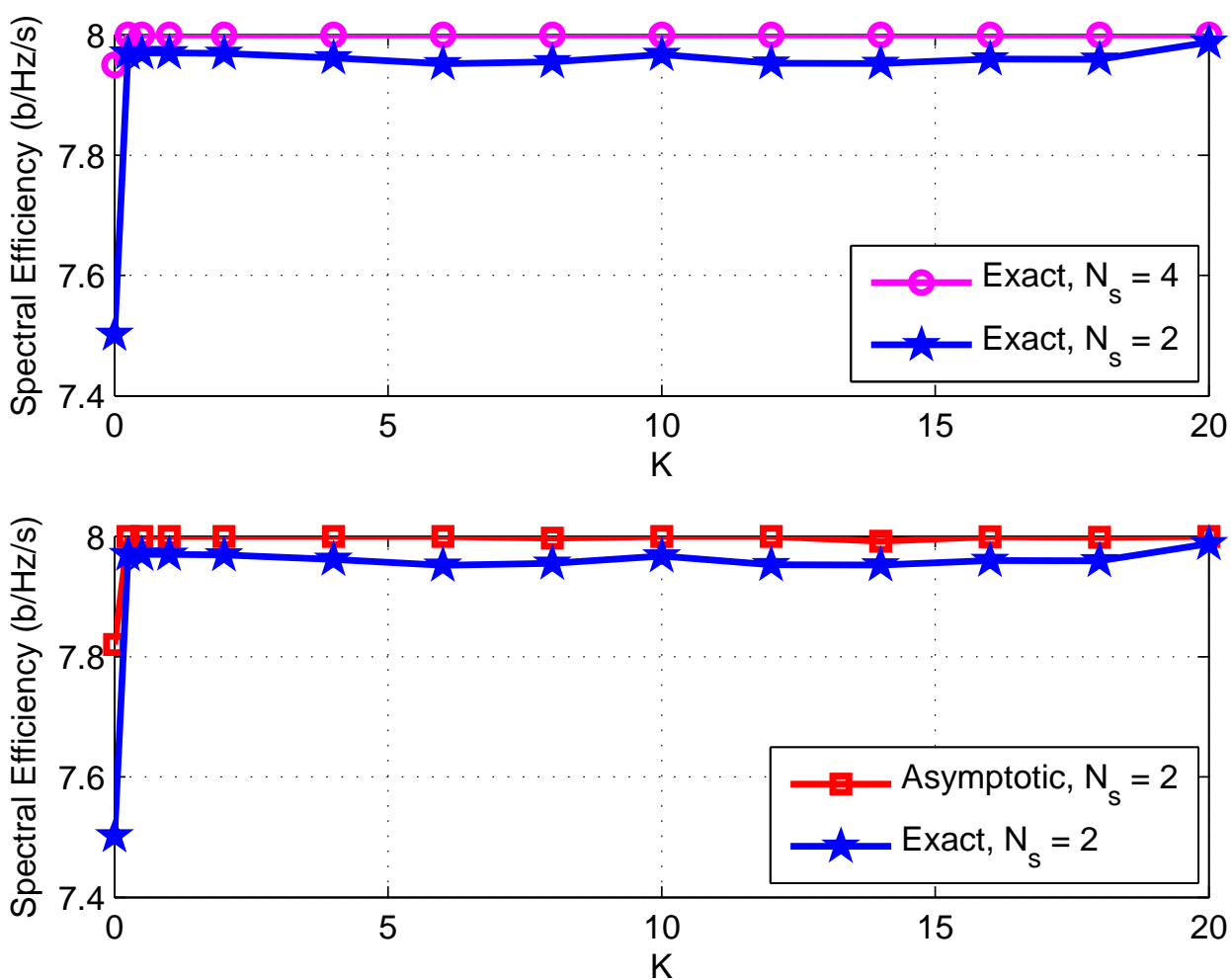

Fig. 1: Spectral efficiency vs. Rice factor $K$ for the channel in (10) with $N_{\mathrm{t}}=N_{\mathrm{r}}=4, \mathrm{SNR}=15 \mathrm{~dB}$, and QPSK.

achieving precoder [53]. Also, the performance of the maximum ratio transmission precoder from [54] is simulated, denoted by "MRT precoder". We substitute the final precoder matrices obtained by different designs into (14) to evaluate the ergodic spectral efficiency. For Algorithm 1, both $N_{\mathrm{s}}=4$ and $N_{\mathrm{s}}=2$ are considered, and despite their enormous computational gap (cf. Table IV) the difference in performance is minor. Both precoders hug the capacity up to the point where the QPSK cardinality becomes insufficient. The proposed design gains many $\mathrm{dB}$ over an unprecoded transmitter, the capacity-achieving precoder applied with QPSK, and the MRT precoder. It is observed in Fig. 2 that, when SNR is low, the performance of the MRT and the capacity-achieving precoders is close to that of the proposed design. This is because the MRT precoder is actually a beamformer and, in the low SNR regime, the beamforming design is near-optimal for both Gaussian input and finite-alphabet inputs [8]. However, as the SNR increases, the beamforming design results in a pronounced performance loss, as shown in Fig. 2.

Fig. 3 contrasts the spectral efficiency given by the asymptotic expression in (23) with the exact form in (14) for the precoders obtained by Algorithm 1 with $N_{\mathrm{s}}=2$. The channel model is the same as for Fig. 


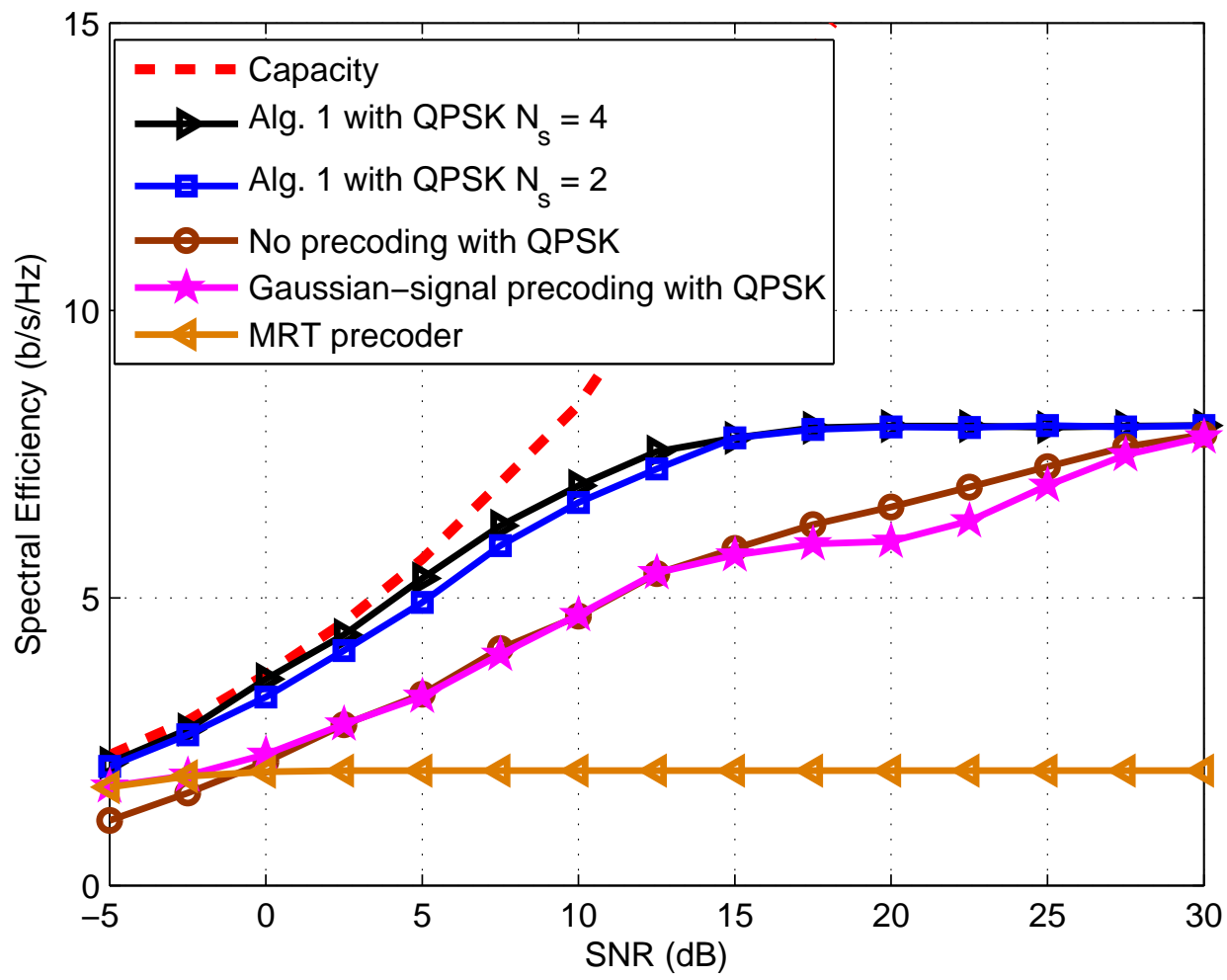

Fig. 2: Spectral efficiency vs. SNR for the 3GPP SCM (urban scenario, half-wavelength antenna spacing, $36 \mathrm{~km} / \mathrm{h}$ ) for different precoder designs with $N_{\mathrm{t}}=N_{\mathrm{r}}=4$ and QPSK.

2. We observe from Fig. 3 that even for a small number of antennas, the asymptotic spectral efficiency in (23) is close to the exact spectral efficiency.

Fig. 4 illustrates the rapid convergence of Algorithm 1 for $N_{\mathrm{s}}=2$ and $N_{\mathrm{s}}=4$ at $\mathrm{SNR}=5 \mathrm{~dB}$. The channel model is the same as for Fig. 2 ,

Figs. 5 and 6 present further results for $N_{\mathrm{t}}=N_{\mathrm{r}}=32$ with QPSK and 16-QAM, respectively. We set $N_{\mathrm{s}}=4$ for the former and $N_{\mathrm{s}}=2$ for the latter. When $N_{\mathrm{t}}=32$, the computational complexity of calculating the ergodic spectral efficiency in (14) scales with $4^{64}$ and $16^{64}$ for QPSK and 16-QAM, respectively, which is prohibitive. Algorithm 1, in contrast, can be executed with very satisfactory performance.

Finally, Figs. 7 and 8 show the convergence of Algorithm 1 at different SNRs for the same settings as in Figs. 5 and 6, respectively. In all cases, convergence occurs within 10 iterations.

\section{CONCLUSION}

With a proper design of $\mathrm{U}_{\mathrm{B}}, \Lambda_{\mathrm{B}}$, and $\mathrm{V}_{\mathrm{B}}$, it is possible to achieve a satisfactory tradeoff between the need to feed into the channel mixings of multiple finite-cardinality signals and the computational 


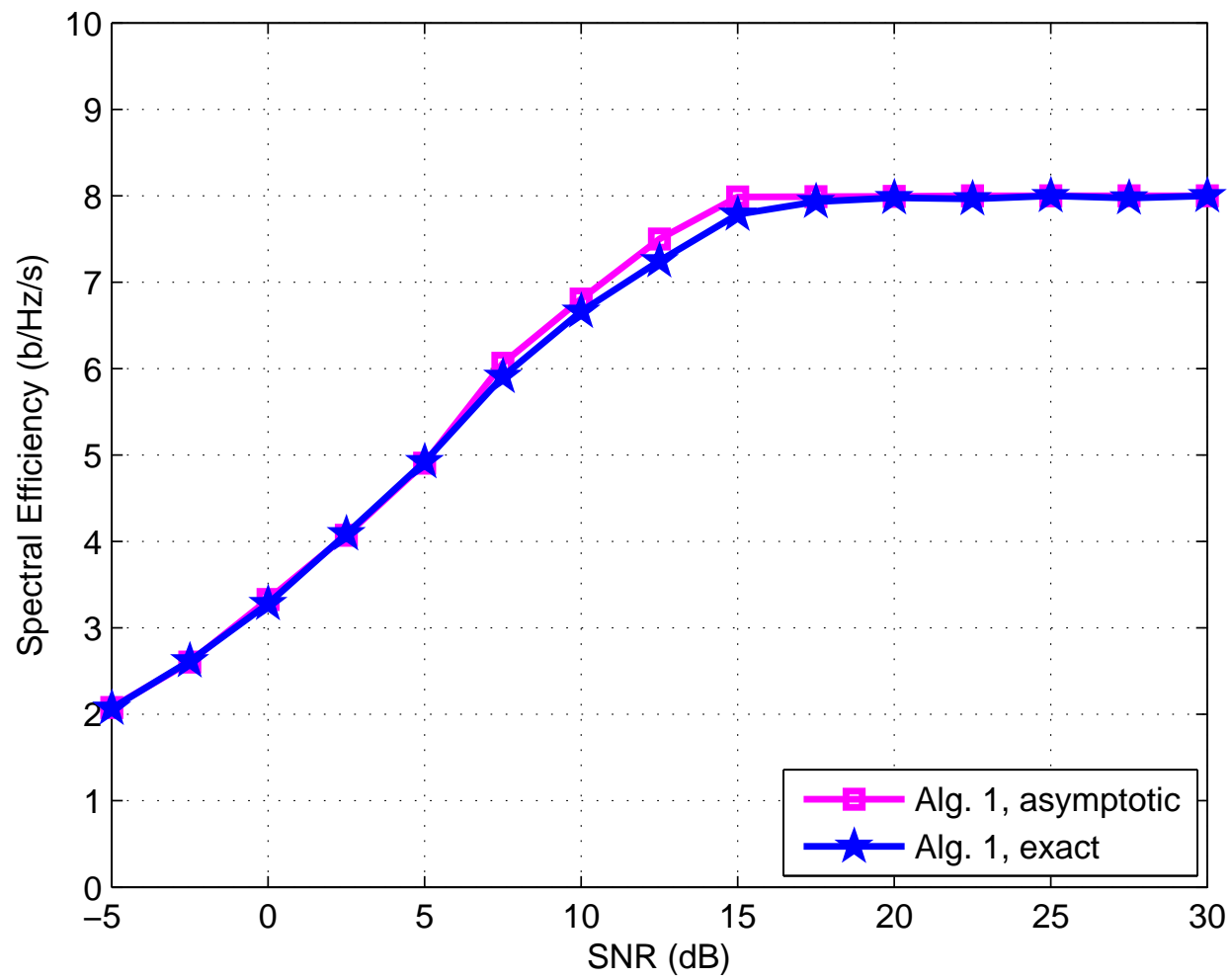

Fig. 3: Asymptotic and exact spectral efficiency vs. SNR for the 3GPP SCM (urban scenario, half-wavelength antenna spacing, $36 \mathrm{~km} / \mathrm{h}$ ) with $N_{\mathrm{t}}=N_{\mathrm{r}}=4, N_{\mathrm{s}}=2$, and QPSK.

complexity of exploring all possible such mixings. Building on this idea, an algorithm has been proposed that-under the 3GPP SCM channel model-exhibits very good performance with orders-of-magnitude less complexity than complete-search solutions while needing only statistical CSI at the transmitter.

The proposed algorithm utilizes the first- and second-order channel statistics $\overline{\mathbf{H}}, \mathbf{U}_{\mathrm{R}}, \tilde{\mathbf{G}}$, and $\mathbf{U}_{\mathrm{T}}$. For growing Rice factors, as the channel becomes progressively deterministic, statistical and instantaneous CSI become equivalent; naturally then, the algorithm converges to instantaneous-CSI solutions. Similarly, if $\mathbf{U}_{\mathrm{R}}=\mathbf{I}_{N_{\mathrm{r}}}$ and $\mathbf{U}_{\mathrm{T}}=\mathbf{I}_{N_{\mathrm{t}}}$, then the algorithm can embrace precoder designs with estimated CSI, where $\overline{\mathbf{H}}$ represents the estimated channel and $\mathbf{G}$ reflects the power of the estimation error.

\section{APPENDIX A}

\section{PROOF OF PROPOSITION 1}

From (14), the ergodic mutual information can be expressed as $I(\mathbf{x} ; \mathbf{y})=\mathcal{F}-N_{\mathrm{r}}$ with $\mathcal{F}=-E_{\mathbf{y}, \mathbf{H}}[\log Z$ $(\mathbf{y}, \mathbf{H})]$ and $Z(\mathbf{y}, \mathbf{H})=E_{\mathbf{x}}\left[e^{-\|\mathbf{y}-\mathbf{H x}\|^{2}}\right]$. The expectations over $\mathbf{y}$ and $\mathbf{H}$ are generally difficult to analyze 


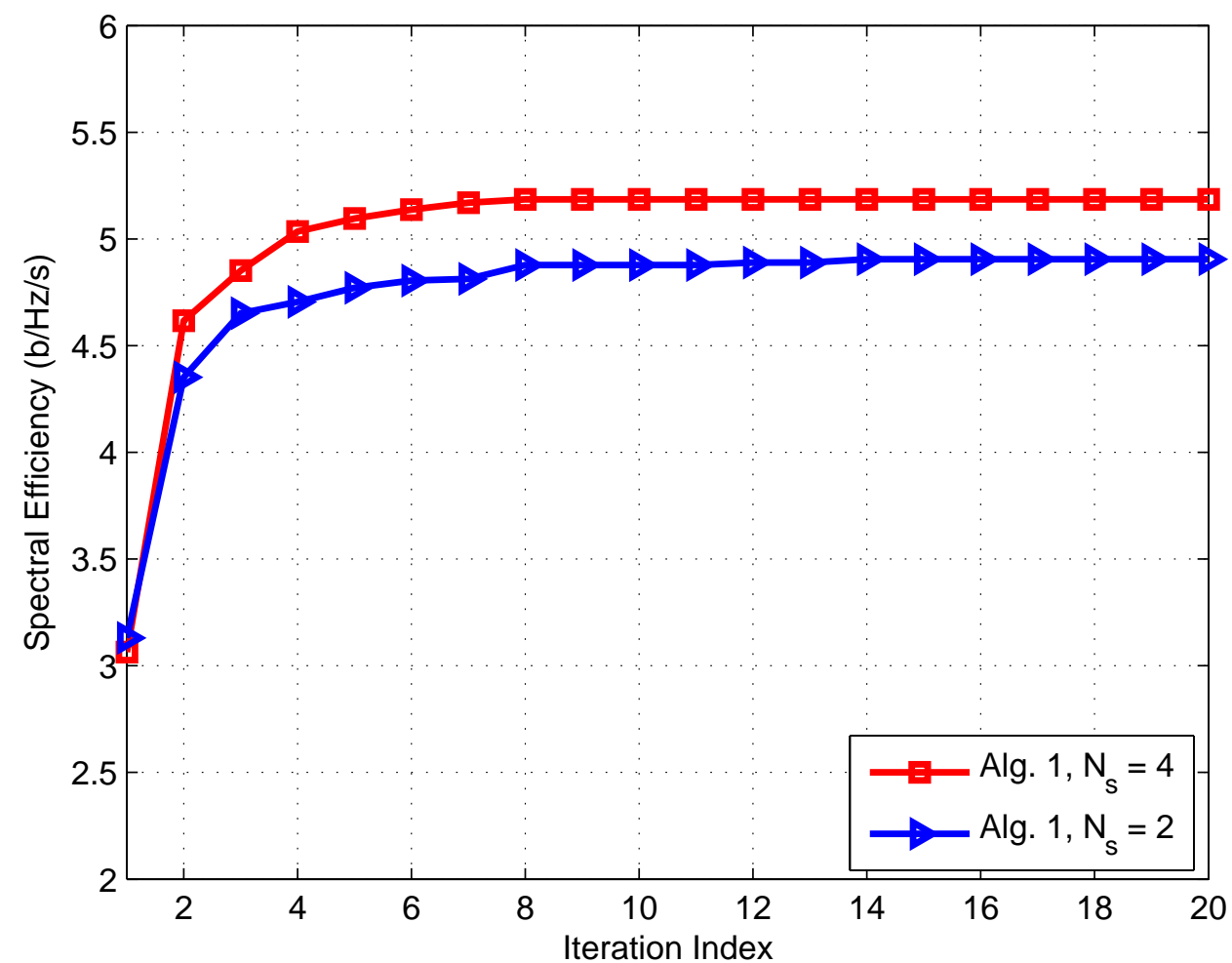

Fig. 4: Convergence of Algorithm 1 for the 3GPP SCM (urban scenario, half-wavelength antenna spacing, $36 \mathrm{~km} / \mathrm{h}$ ) with $N_{\mathrm{t}}=N_{\mathrm{r}}=4$ and QPSK.

because of the logarithm. However, these difficulties can be circumvented by rewriting $\mathcal{F}$ as [46, (2.6)]

$$
\mathcal{F}=-\lim _{\tau \rightarrow 0} \frac{\partial}{\partial \tau} \log E_{\mathbf{y}, \mathbf{H}}\left[Z^{\tau}(\mathbf{y}, \mathbf{H})\right] .
$$

This reformulation allows evaluating $E_{\mathbf{y}, \mathbf{H}}\left[Z^{\tau}(\mathbf{y}, \mathbf{H})\right]$ for integer $\tau$, and subsequently for $\tau$ in the vicinity of 0 . This so-called replica method [47] has been widely adopted in statistical physics [46] and information theory [35], [48]-[53], [55].

The calculation of $\mathcal{F}$ via the replica method consists of the following three steps. First, we introduce $\tau$ IID replicated symbols $\mathbf{x}^{(\alpha)}$, for $\alpha=0,1, \ldots, \tau$, and then, we compute the expectations over $\mathbf{y}$ and $\mathbf{H}$ by repeatedly using the Gaussian integral 4 Second, we simplify the obtained expression for $E_{\mathbf{y}, \mathbf{H}}\left[Z^{\tau}(\mathbf{y}, \mathbf{H})\right]$ by assuming that the covariance matrices of the replicas are in symmetry form [46, Section 2.3]. Finally, we

${ }^{4}$ Let $\mathbf{S} \in \mathbb{C}^{m \times n}, \mathbf{A}_{1} \in \mathbb{C}^{m \times n}$, and $\mathbf{A}_{2} \in \mathbb{C}^{m \times n}$ be complex matrices and $\mathbf{A}_{3} \in \mathbb{C}^{n \times n}$ and $\mathbf{A}_{4} \in \mathbb{C}^{m \times m}$ positive definite matrices, respectively. Then, the following equality holds [49], [35 Lemma 1]:

$$
\int D \mathbf{S} e^{-\operatorname{tr}\left(\mathbf{A}_{3} \mathbf{S}^{H} \mathbf{A}_{4} \mathbf{S}+\mathbf{A}_{1}^{H} \mathbf{S}-\mathbf{S}^{H} \mathbf{A}_{2}\right)}=\frac{1}{\operatorname{det}\left(\mathbf{A}_{3} \otimes \mathbf{A}_{4}\right)} e^{-\operatorname{tr}\left(\mathbf{A}_{3}^{-1} \mathbf{A}_{1}^{H} \mathbf{A}_{4}^{-1} \mathbf{A}_{2}\right) .}
$$




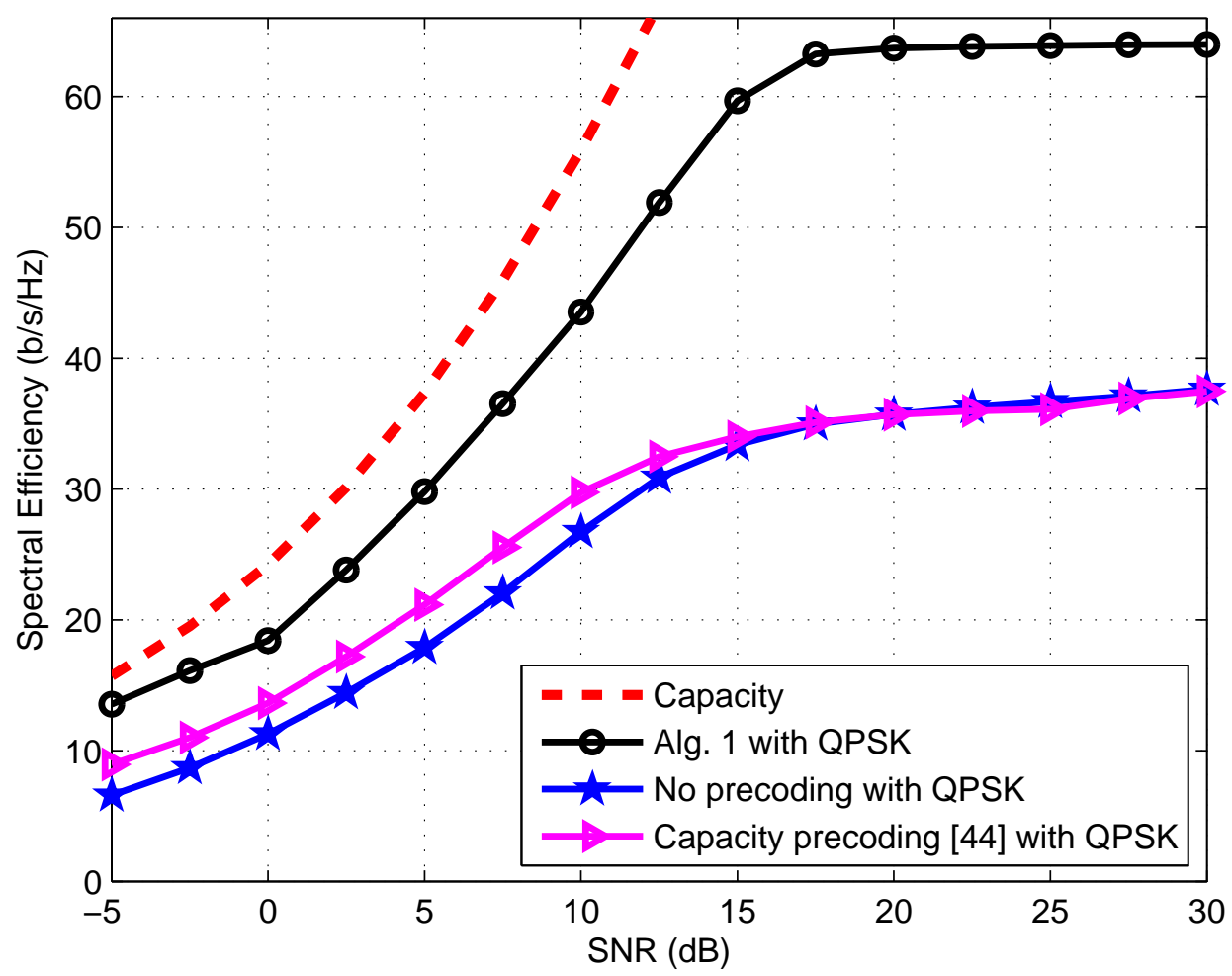

Fig. 5: Spectral efficiency vs. SNR for the 3GPP SCM (urban scenario, half-wavelength antenna spacing, $36 \mathrm{~km} / \mathrm{h}$ ) for different precoder designs with $N_{\mathrm{t}}=N_{\mathrm{r}}=32, N_{\mathrm{s}}=4$, and QPSK.

compute the remaining integrals by using the saddle-point method (or the method of steepest descent [46, Section 2.2.4]), and explicitly find the saddle points at $\tau \rightarrow 0$. In the following, we limit our presentation to the main steps, since analogous calculations can be found in several earlier works [35], [48]-[53], [55].

\section{Step 1 (Replica analysis):}

To compute $E_{\mathbf{y}, \mathbf{H}}\left[Z^{\tau}(\mathbf{y}, \mathbf{H})\right]$ it is useful to introduce $\tau$ IID replicated symbols $\mathbf{x}^{(\alpha)}$, for $\alpha=0,1, \ldots, \tau$, yielding

$$
E_{\mathbf{y}, \mathbf{H}}\left[Z^{r}(\mathbf{y}, \mathbf{H})\right]=E_{\mathbf{H}, \mathbf{X}}\left[\int D \mathbf{y} \prod_{\alpha=0}^{\tau} e^{-\left\|\mathbf{y}-\mathbf{H x}^{(\alpha)}\right\|^{2}}\right]
$$

where $\mathbf{X}=\left[\mathbf{x}^{(0)} \mathbf{x}^{(1)} \ldots \mathbf{x}^{(\tau)}\right]$. The indices $\alpha$ represent different so-called replicas of the system. The integral with respect to $\mathbf{y}$ in (74) can be evaluated using the Gaussian integral. Then, to disentangle $\mathbf{H}$ and $\mathbf{X}$, we introduce a set of random variables $v_{n m}^{(\alpha)}=[\mathbf{W}]_{n m}[\tilde{\mathbf{G}}]_{n m} \mathbf{u}_{\mathrm{T}, m}^{H} \mathbf{x}^{(\alpha)}$. Given $[\tilde{\mathbf{G}}]_{n m}, \mathbf{u}_{\mathrm{T}, m}^{H}$, and $\mathbf{x}^{(\alpha)}$, it is easily found that the $v_{n m}^{(\alpha)}$ are Gaussian with zero-mean and covariance $E\left[v_{n m}^{(\alpha) H} v_{n m}^{(\beta)}\right]=$ 


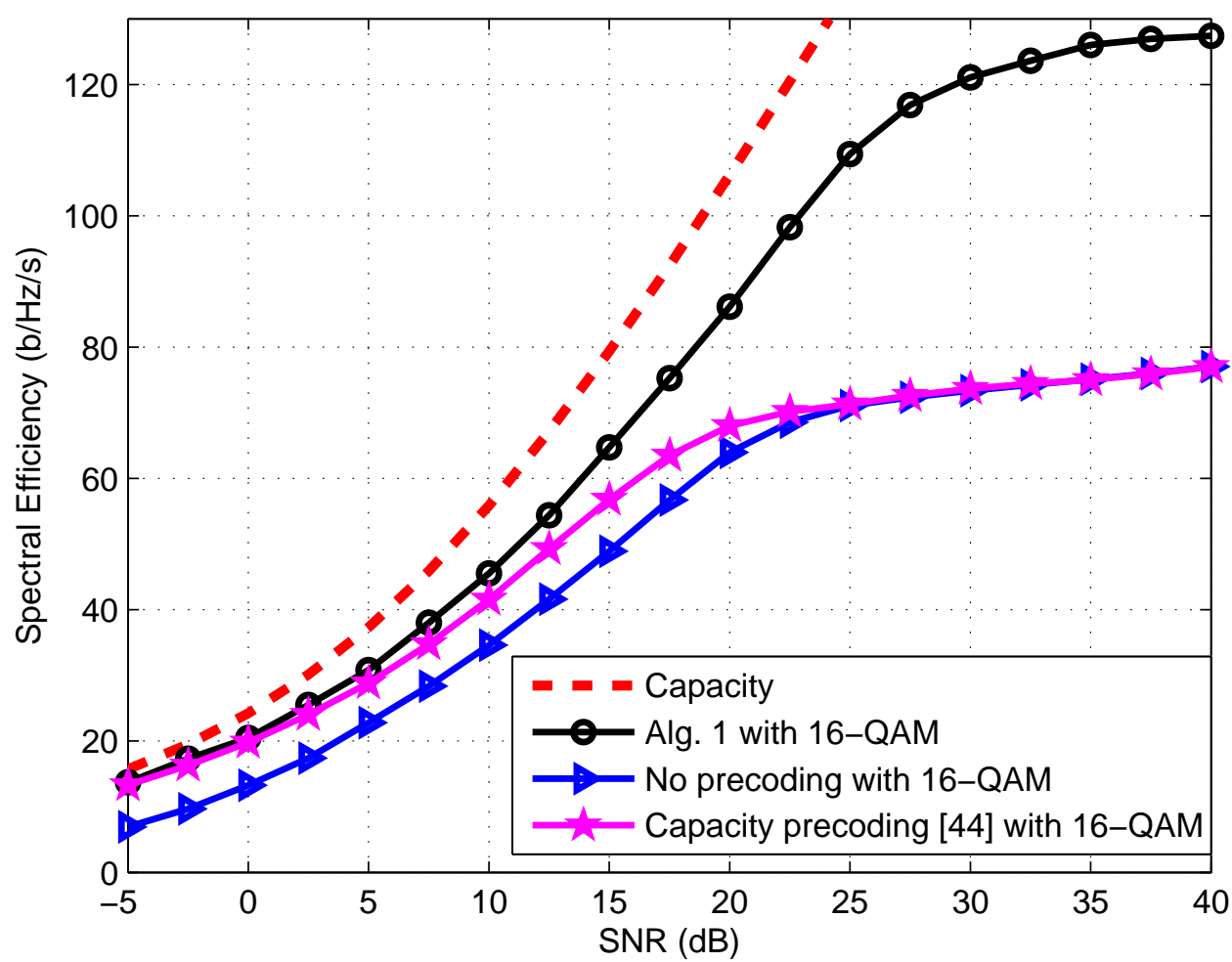

Fig. 6: Spectral efficiency vs. SNR for the 3GPP SCM (urban scenario, half-wavelength antenna spacing, $36 \mathrm{~km} / \mathrm{h}$ ) for different precoder designs with $N_{\mathrm{t}}=N_{\mathrm{r}}=32, N_{\mathrm{s}}=2$, and 16-QAM.

$[\mathbf{G}]_{n m}\left(\mathbf{x}^{(\alpha)}\right)^{H} \mathbf{T}_{m} \mathbf{x}^{(\beta)}=Q_{n m}^{(\alpha, \beta)}, \forall \alpha, \beta$, where $\mathbf{T}_{m}=\mathbf{u}_{\mathrm{T}, m} \mathbf{u}_{\mathrm{T}, m}^{H}$. Then, we insert an identity that captures all combinations of the replicas

$$
1=\int \prod_{n, m} \prod_{0 \leq \alpha \leq \beta}^{r} \delta\left([\mathbf{G}]_{n m} \mathbf{x}^{(\alpha) H} \mathbf{T}_{m} \mathbf{x}^{(\beta)}-Q_{n m}^{(\alpha, \beta)}\right)
$$

into (74). Let us define $\mathbf{Q}_{n m} \in \mathbb{C}^{(\tau+1) \times(\tau+1)}$ with $\left[\mathbf{Q}_{n m}\right]_{\alpha \beta}=Q_{n m}^{(\alpha, \beta)}, \mathbb{Q}=\left\{\mathbf{Q}_{n m}\right\}_{\forall n, m}, v_{n}^{(\alpha)}=\sum_{m} v_{n m}^{(\alpha)}$, and $\mathcal{V}=\left\{v_{n m}^{(\alpha)}\right\}$. Then, (74) can be written as

$$
E_{\mathbf{y}, \mathbf{H}}\left[Z^{\tau}(\mathbf{y}, \mathbf{H})\right]=\int e^{\mathcal{S}(\mathbb{Q})} d \mu(\mathbb{Q})
$$

where

$$
\begin{aligned}
& \mathcal{S}^{(\tau)}(\mathbb{Q})=\log \int D \mathbf{y} E_{\mathcal{V}}\left[\prod_{\tau} e^{\left.-\left\|\mathbf{y}-\sum_{n} v_{n}^{(\alpha)} \mathbf{u}_{\mathrm{R} n}-\overline{\mathbf{H}} \mathbf{x}^{(\alpha)}\right\|^{2}\right]}\right. \\
& \mu^{(\tau)}(\mathbb{Q})=E_{\mathbf{X}}\left[\prod_{n, m} \prod_{0 \leq \alpha \leq \beta}^{r} \delta\left([\mathbf{G}]_{n m} \mathbf{x}^{(\alpha) H} \mathbf{T}_{m} \mathbf{x}^{(\beta)}-Q_{n m}^{(\alpha, \beta)}\right)\right] .
\end{aligned}
$$

The integral in (76) can now be estimated by applying the saddle-point method. Therefore, we are left with the evaluation of $\mathcal{S}^{(\tau)}(\mathbb{Q})$ and $\mu^{(\tau)}(\mathbb{Q})$ which can be computed by applying the techniques in $[35$, 


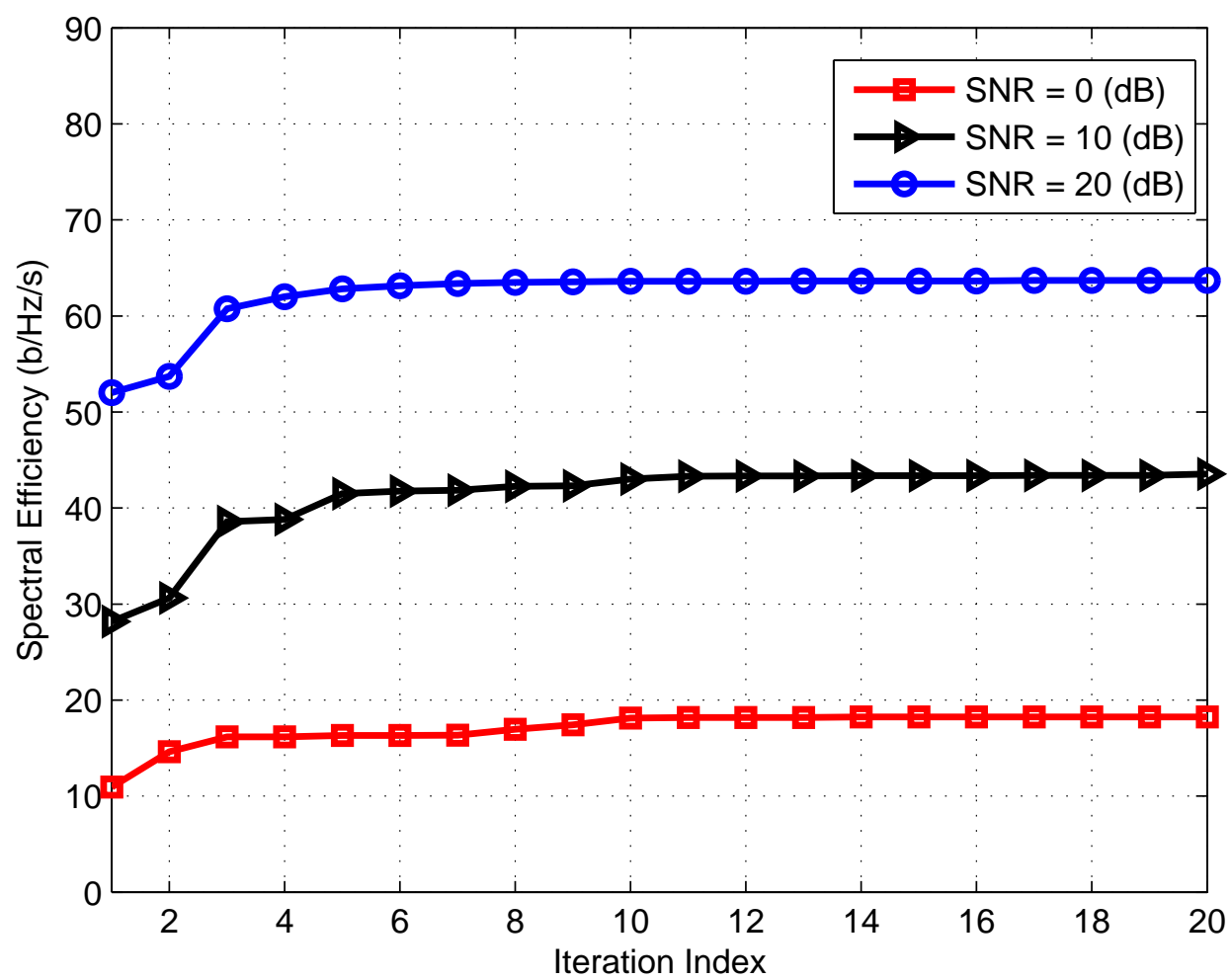

Fig. 7: Convergence of Algorithm 1 for the 3GPP SCM (urban scenario, half-wavelength antenna spacing, $36 \mathrm{~km} / \mathrm{h}$ ) for different precoder designs with $N_{\mathrm{t}}=N_{\mathrm{r}}=32, N_{\mathrm{s}}=4$, and QPSK.

Appendix A]. Specifically, the evaluation of $\mu^{(\tau)}(\mathbb{Q})$ is exactly identical to that in [35, (35)], whereas for $\mathcal{S}^{(\tau)}(\mathbb{Q})$, additional manipulations for dealing with $\overline{\mathbf{H}} \mathbf{x}^{(\alpha)}$ in (77) are required.

Because of the Gaussian nature of $v_{n m}^{(\alpha)}$, we can calculate the expectation over $\mathcal{V}$ after integrating over $\mathbf{y}$ in (77). Meanwhile, we apply the inverse Laplace transform of $\delta(\cdot) 5$ to (78) by introducing auxiliary variables $\tilde{\mathbf{Q}}_{n m} \in \mathbb{C}^{(\tau+1) \times(\tau+1)}$ and letting $\tilde{\mathbb{Q}}=\left\{\tilde{\mathbf{Q}}_{n m}\right\}_{\forall n, m}$. The remaining integrals over $(\mathbb{Q}, \tilde{\mathbb{Q}})$ can be evaluated via the saddle point method yielding

$$
\mathcal{F}=-\lim _{\tau \rightarrow 0} \frac{\partial}{\partial \tau} \max _{\mathbb{Q}, \tilde{\mathbb{Q}}}\left\{\mathcal{F}^{(\tau)}\right\}
$$

with $\mathcal{F}^{(\tau)}=\mathcal{S}^{(\tau)}+\mathcal{J}^{(\tau)}$, where

$$
\mathcal{S}^{(\tau)}=-N_{\mathrm{r}} \log (\tau+1)-\log \operatorname{det}\left(\mathbf{I}_{N_{\mathrm{r}}(\tau+1)}+\mathbf{Q} \boldsymbol{\Sigma} \otimes \mathbf{R}\right)
$$

${ }^{5}$ The inverse Laplace transform of the $\delta$-function is given by [46, (5.140)]

$$
\delta(x)=\frac{1}{2 \pi j} \int_{-j \infty+t}^{j \infty+t} e^{\tilde{Q} x} d \tilde{Q}, \forall t \in \mathbb{R} .
$$




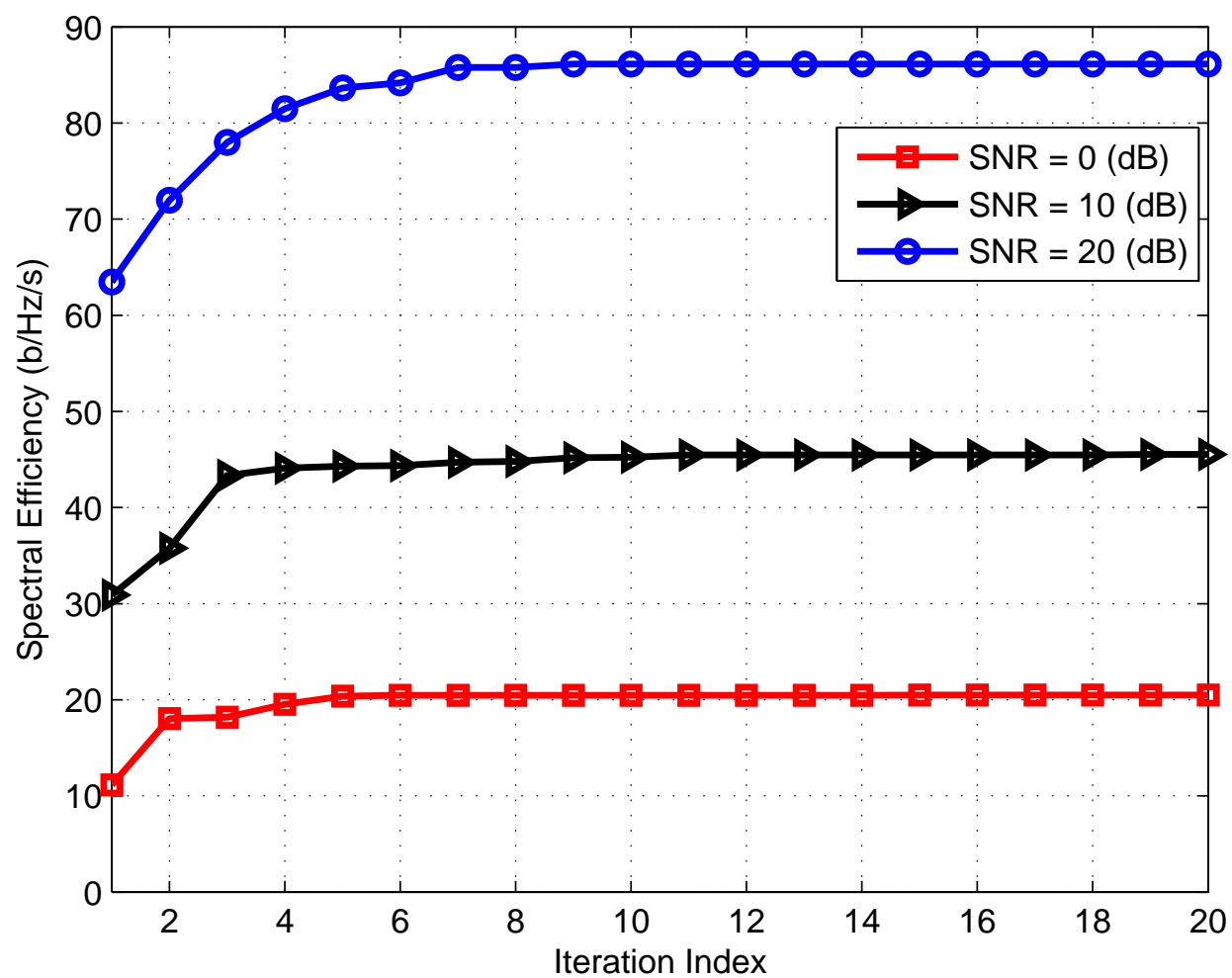

Fig. 8: Convergence of Algorithm 1 for the 3GPP SCM (urban scenario, half-wavelength antenna spacing, $36 \mathrm{~km} / \mathrm{h}$ ) for different precoder designs with $N_{\mathrm{t}}=N_{\mathrm{r}}=32, N_{\mathrm{s}}=2$, and 16-QAM.

$$
\begin{aligned}
\mathcal{J}^{(\tau)} & =\max _{\tilde{\mathbb{Q}}}\left\{\sum_{n, m} \operatorname{tr}\left(\tilde{\mathbf{Q}}_{n m} \mathbf{Q}_{n m}\right)-\log E_{\mathbf{X}}\left[e^{\sum_{m} \operatorname{tr}\left(\sum_{n}[\mathbf{G}]_{n m} \tilde{\mathbf{Q}}_{n m} \mathbf{X}^{H} \mathbf{T}_{m} \mathbf{X}\right)}\right.\right. \\
& \left.\cdot e^{\left.\operatorname{vec}(\overline{\mathbf{V}})^{H}\left[(\mathbf{Q} \otimes \mathbf{R})^{-1}\left(\left(\mathbf{Q} \boldsymbol{\Sigma} \otimes \mathbf{R}+\mathbf{I}_{N_{\mathrm{r}}(\tau+1)}\right)^{-1}-\mathbf{I}_{N_{\mathrm{r}}(\tau+1)}\right)\right] \operatorname{vec}(\overline{\mathbf{V}})\right]}\right\},
\end{aligned}
$$

$\boldsymbol{\Sigma}=\mathbf{I}_{\tau+1}-\frac{1}{(\tau+1)} \mathbf{1} \mathbf{1}^{T}, \mathbf{Q} \otimes \mathbf{R}=\sum_{n}\left(\sum_{m} \mathbf{Q}_{n m}\right) \otimes \mathbf{R}_{n}, \mathbf{R}_{n}=\mathbf{u}_{\mathrm{R}, n} \mathbf{u}_{\mathrm{R}, n}^{H}$, and $\overline{\mathbf{V}}=\overline{\mathbf{H}} \mathbf{X}$. For the case with no LOS, the last exponential term in the last line of (81) disappears [35, (39)]. Hence, the LOS makes a nontrivial difference.

\section{Step 2 (Replica symmetry assumption):}

The extremum over $(\mathbb{Q}, \tilde{\mathbb{Q}})$ in $(79)$ can be obtained by seeking the point of zero gradient, yielding a set of saddle-point equations. However, explicit expressions for the saddle points are not forthcoming. Therefore, we assume that the saddle points exhibit the replica symmetry (RS) form [35, (41) and (42)] $\mathbf{Q}_{n m}=q_{n m} \mathbf{1 1}^{T}+\left(c_{n m}-q_{n m}\right) \mathbf{I}_{\tau+1}$ and $\tilde{\mathbf{Q}}_{n m}=\tilde{q}_{n m} \mathbf{1 1 ^ { T }}+\left(\tilde{c}_{n m}-\tilde{q}_{n m}\right) \mathbf{I}_{\tau+1}$. Based on RS, $q_{n m}, c_{n m}, \tilde{q}_{n m}$, and $\tilde{c}_{n m}$ are four parameters that need to be determined. 
Step 3 (Saddle point):

$q_{n m}, c_{n m}, \tilde{q}_{n m}$, and $\tilde{c}_{n m}$ can be obtained by inserting the RS into $\mathcal{F}^{(\tau)}$ and equating the partial derivatives of the corresponding $\mathcal{F}^{(\tau)}$ to zero. In this case, it can be verified that $\tilde{c}_{n m}=0$. Let $\gamma_{n m}=\tilde{q}_{n m}$ and $\psi_{n m}=\left(c_{n m}-q_{n m}\right) /[\mathbf{G}]_{n m}$. Finally, at $\tau=0, \mathcal{F}$ can be expressed as

$$
\mathcal{F} \simeq I(\mathbf{x} ; \mathbf{z} \mid \sqrt{\mathbf{\Xi}})+\log \operatorname{det}\left(\mathbf{I}_{N_{\mathrm{r}}}+\mathbf{R}\right)-\sum_{n, m} \gamma_{n m}[\mathbf{G}]_{n m} \psi_{n m}+N_{\mathrm{r}}
$$

where $\boldsymbol{\Xi}=\mathbf{T}+\overline{\mathbf{H}}^{H}(\mathbf{I}+\mathbf{R})^{-1} \overline{\mathbf{H}}$ with $\mathbf{T}=\sum_{m}\left(\sum_{n} \gamma_{n m}\right) \mathbf{T}_{m}, \mathbf{R}=\sum_{n}\left(\sum_{m} \psi_{n m}\right) \mathbf{R}_{n}$. Equating the partial derivatives of $\mathcal{F}$ over $\gamma_{n m}$ and $\psi_{n m}$, we obtain $\gamma_{n m}=\gamma_{m}$ and $\psi_{n m}=\psi_{n}$ as given in (21). Note that, since $\gamma_{n m}$ and $\psi_{n m}$ are independent of $m$ and $n$, respectively, we have replaced them with $\gamma_{m}$ and $\psi_{n}$ in (21). Using $I(\mathbf{x} ; \mathbf{y})=\mathcal{F}-N_{\mathrm{r}}$ as given at the beginning of this appendix, we finally obtain Proposition 1.

\section{APPENDIX B}

\section{ProOf of Proposition 2}

Consider the optimization problem

$$
\begin{array}{ll}
\max _{\mathbf{B}} & I_{\text {asy }}(\mathbf{x} ; \mathbf{y}) \\
\text { s.t. } & \operatorname{tr}\left(\mathbf{B B}^{H}\right) \leq P .
\end{array}
$$

The equivalent channel matrix $\Xi$ in $(18)$ is a function of the precoder $\mathbf{B}$ through the coupled equations (19)-(21). Thus, the derivation in [20, App. A] that requires the channel matrix to be independent of the precoder cannot be applied directly here.

To solve (83), we establish the Lagrangian function for (83) in terms of the precoder $\mathbf{B}$ as

$$
g(\mathbf{B})=-I_{\text {asy }}(\mathbf{x} ; \mathbf{y})+\kappa\left[\operatorname{tr}\left(\mathbf{B B}^{H}\right)-P\right]
$$

where $\kappa$ is a Lagrange multiplier.

The Karush-Kuhn-Tucker condition [56] dictates that $\nabla_{\mathbf{B}} g(\mathbf{B})=\mathbf{0}$ or, equivalently, that

$$
-\nabla_{\mathbf{B}} I_{\text {asy }}(\mathbf{x} ; \mathbf{y})+\kappa \mathbf{B}=\mathbf{0} \text {. }
$$


For ease of exposition, we define $I_{1}(\mathbf{B})=I(\mathbf{x} ; \mathbf{z})$. For $I_{\text {asy }}(\mathbf{x} ; \mathbf{y})$ in $(24)$, the parameters affected by the perturbation of $\mathbf{B}$ are $\left\{I_{1}(\mathbf{B}), \gamma_{m}, \psi_{n}\right\}$. According to the chain rule, the gradient of $I_{\text {asy }}(\mathbf{x} ; \mathbf{y})$ with respect to $\mathbf{B}$ is given by

$$
\nabla_{\mathbf{B}} I_{\text {asy }}(\mathbf{x} ; \mathbf{y})=\frac{\partial I_{\text {asy }}(\mathbf{x} ; \mathbf{y})}{\partial I_{1}(\mathbf{B})} \nabla_{\mathbf{B}} I_{1}(\mathbf{B})+\log _{2} e\left(\sum_{m=1}^{N_{\mathrm{r}}} \frac{\partial I_{\text {asy }}(\mathbf{x} ; \mathbf{y})}{\partial \gamma_{m}} \nabla_{\mathbf{B}} \gamma_{m}+\sum_{n=1}^{N_{\mathrm{t}}} \frac{\partial I_{\mathrm{asy}}(\mathbf{x} ; \mathbf{y})}{\partial \psi_{n}} \nabla_{\mathbf{B}} \psi_{n}\right) .
$$

The relationship between the channel $\Xi$ in $(18)$ and the precoder $\mathbf{B}$ is determined by the parameters $\gamma_{m}$ and $\psi_{n}$ in (21). Hence, when calculating the first term on the right-side of (86), $\Xi$ is regarded as independent of B. Also, from the definitions of $\gamma_{m}$ and $\psi_{n}$ in Appendix $\mathbf{A}$, we have that

$$
\frac{\partial I_{\text {asy }}(\mathbf{x} ; \mathbf{y})}{\partial \gamma_{m}}=0, \frac{\partial I_{\text {asy }}(\mathbf{x} ; \mathbf{y})}{\partial \psi_{n}}=0 \text {. }
$$

As a result, based on (85)-(87) and [36, (22)], the optimal precoder should satisfy the condition

$$
\kappa \mathbf{B}=\Xi \mathrm{B} \Omega
$$

Using the eigenvalue decomposition $\Omega=\mathrm{U}_{\Omega} \Lambda_{\Omega} \mathrm{U}_{\Omega}^{H}$, we can rewrite (88) as

$$
\kappa \mathbf{U}_{\Xi}^{H} \mathbf{B U}_{\Omega}=\Lambda_{\Xi} \mathbf{U}_{\Xi}^{H} \mathbf{B U}_{\Omega} \Lambda_{\Omega}
$$

Define $\mathbf{Q}=\mathbf{U}_{\Xi}^{H} \mathbf{B} \mathbf{U}_{\boldsymbol{\Omega}}$. Then, we have

$$
\kappa \mathbf{Q}=\operatorname{diag}\left(\boldsymbol{\Lambda}_{\boldsymbol{\Xi}}\right) \operatorname{diag}\left(\boldsymbol{\Lambda}_{\Omega}\right)^{T} \odot \mathbf{Q}
$$

which is equivalent to

$$
\kappa[\mathbf{Q}]_{m n}=\left[\boldsymbol{\Lambda}_{\boldsymbol{\Xi}}\right]_{m m}\left[\boldsymbol{\Lambda}_{\boldsymbol{\Omega}}\right]_{n n}[\mathbf{Q}]_{m n}
$$

The eigenvalues of $\boldsymbol{\Xi}$ and $\Omega$ are distinct with probability one. Therefore, the equality $\kappa=\left[\boldsymbol{\Lambda}_{\boldsymbol{\Xi}}\right]_{m m}\left[\boldsymbol{\Lambda}_{\boldsymbol{\Omega}}\right]_{n n}$ can be satisfied for at most $N_{\mathrm{t}}$ pairs of $(m, n)$, each corresponding to different $m$ and $n$. For other pairs of $(m, n),[\mathbf{Q}]_{m n}=0$ so that $(91)$ can hold. As a result, $\mathbf{Q}$ has at most one nonzero entry in each row and in each column. Thus, $\mathrm{Q}$ can be written as

$$
\mathrm{Q}=\Lambda \Pi
$$

where $\Lambda$ is diagonal and $\Pi$ is a permutation matrix. Recalling the definition of $Q$, the optimal precoder is $\mathbf{B}=\mathbf{U}_{\Xi} \Lambda \Pi \mathbf{U}_{\Omega}^{H}$. 


\section{REFERENCES}

[1] A. Lozano, A. M. Tulino, and S. Verdú, "Optimum power allocation for parallel Gaussian channels with arbitrary input distributions," IEEE Trans. Inf. Theory, vol. 52, pp. 3033-3051, Jul. 2006.

[2] A. Lozano, A. M. Tulino, and S. Verdú, "Optimum power allocation for multiuser OFDM with arbitrary signal constellation," IEEE Trans. Commun., vol. 56, pp. 828-837, May 2008.

[3] C. Xiao, Y. R. Zheng, and Z. Ding, "Globally optimal linear precoders for finite alphabet signals over complex vector Gaussian channels," IEEE Trans. Signal Process., vol. 59, pp. 3301-3314, Jul. 2011.

[4] C. Xiao and Y. R. Zheng, "On the mutual information and power allocation for vector Gaussian channels with finite discrete inputs," in Proc. IEEE Global. Telecommun. Conf. (GLOBECOM 2008), New Orleans, USA, Dec. 2008, pp. 1-5.

[5] — - "Transmit precoding for MIMO systems with partial CSI and discrete-constellation inputs," in Proc. IEEE Int. Telecommun. Conf. (ICC 2009), Dresden, Germany, Jun. 2009, pp. 1-5.

[6] M. Payaró and D. P. Palomar, "On optimal precoding in linear vector Gaussian channels with arbitrary inputs distribution," in Proc. IEEE Int. Symp. Inform. Theory (ISIT 2009), Seoul, Korea, Jun. 2009, pp. 1085-1089.

[7] M. Lamarca, "Linear precoding for mutual information maximization in MIMO systems," in Proc. Int. Symp. Wireless Commun. Sys. (ISWCS 2009), Siena, Italy, 2009, pp. 1-5.

[8] F. Pérez-Cruz, M. R. D. Rodrigues, and S. Verdú, "MIMO Gaussian channels with arbitrary input: Optimal precoding and power allocation,” IEEE Trans. Inf. Theory, vol. 56, pp. 1070-1084, Mar. 2010.

[9] S. K. Mohammed, E. Viterbo, Y. Hong, and A. Chockalingam, "Precoding by pairing subchannels to increase MIMO capacity with discrete input alphabets," IEEE Trans. Inf. Theory, vol. 57, pp. 4156-4169, Jul. 2011.

[10] T. Ketseoglou and E. Ayanoglu, "Linear precoding for MIMO with LDPC coding and reduced complexity," IEEE Trans. Wireless Commun., vol. 14, pp. 2192-2204, Apr. 2015.

[11] T. Ketseoglou and E. Ayanoglu, "Linear precoding for MIMO channels with QAM constellations and reduced complexity," Online Avaiable: http://arxiv.org/abs/1601.03141v1.

[12] W. Zeng, C. Xiao, and J. Lu, "A low complexity design of linear precoding for MIMO channel with finite alphabet inputs," IEEE Wireless. Commun. Lett., vol. 1, pp. 38-42, Feb. 2012.

[13] A. M. Tulino, A. Lozano, and S. Verdú, "Impact of antenna correlation on the capacity of multiantenna channels," IEEE Trans. Inf. Theory, vol. 51, pp. 2491-2509, Jul. 2005.

[14] A. M. Tulino, A. Lozano, and S. Verdú, "Capacity-achieving input covariance for single-user multi-antenna channels," IEEE Trans. Wireless. Commun., vol. 5, pp. 662-671, Mar. 2006.

[15] X. Gao, B. Jiang, X. Li, A. B. Gershman, and M. R. McKay, "Statistical eigenmode transmission over jointly-correlated MIMO channels," IEEE Trans. Inf. Theory, vol. 55, pp. 3735-3750, Aug. 2009.

[16] J. Wang, S. Jin, X. Gao, K.-K. Wong, and E. Au, "Statistical eigenmode-based SDMA for two-user downlink," IEEE Trans. Signal Process., vol. 60, pp. 5371-5383, Oct. 2012.

[17] J. Zhang, C. K. Wen, S. Jin, X. Gao, and K. K. Wong, "On capacity of large-scale MIMO multiple access channels with distributed sets of correlated antennas," IEEE J. Sel. Areas Commun., vol. 31, pp. 133-148, Feb. 2013.

[18] Y. Wu, S. Jin, X. Gao, M. R. McKay, and C. Xiao, "Transmit designs for the MIMO broadcast channel with statistical CSI," IEEE Trans. Signal Process., vol. 62, pp. 4451-4446, Sep. 2014. 
[19] Y. Wu, R. Schober, D. W. K. Ng, C. Xiao, and G. Caire, "Secure massive MIMO transmission with an active eavesdropper," IEEE Trans. Inform. Theory, vol. 62, pp. 3880-3900,Jul. 2016.

[20] W. Zeng, C. Xiao, M. Wang, and J. Lu, "Linear precoding for finite-alphabet inputs over MIMO fading channels with statistical CSI," IEEE Trans. Signal Process., vol. 60, pp. 3134-3148, Jun. 2012.

[21] J. Salo, G. Del Galdo, J. Salmi, P. Kyösti, M. Milojevic, D. Laselva, and C. Schneider. (2005, Jan.) MATLAB implementation of the 3GPP Spatial Channel Model (3GPP TR 25.996) [Online]. Available: http://www.tkk.fi/Units/Radio/scm/.

[22] W. Weichselberger, M. Herdin, H. Ozcelik, and E. Bonek, "A stochastic MIMO channel model with joint correlation of both link ends," IEEE Trans. Wireless. Commun., vol. 5, pp. 90-100, Jan. 2006.

[23] D.-S. Shiu, G. J. Foschini, M. J. Gans, and J. M. Kahn, "Fading correlation and its effect on the capacity of multielement antenna systems," IEEE Trans. Commun., vol. 48, pp. 502-513, Mar. 2000.

[24] J. P. Kermoal, L. Schumacher, K. I. Pedersen, and P. Mogensen, "A stochastic MIMO radio channel model with experimental validation," IEEE J. Sel. Areas Commun., vol. 20, pp. 1211-1226, Jun. 2002.

[25] C. Xiao, J. Wu, S. Y. Leong, Y. R. Zheng, and K. B. Letaief, "A discrete-time model for triply selective MIMO Rayleigh fading channels," IEEE Trans. Wireless Commun., vol. 3, pp. 1678-1688, Sep. 2004.

[26] A. M. Sayeed, "Deconstructing multiantenna fading channels," IEEE Trans. Signal Process., vol. 50, pp. $2563-2579$, Oct. 2002.

[27] V. Veeravalli, Y. Liang, and A. M. Sayeed, "Correlated MIMO Rayleigh fading channels: Capacity, optimal signaling and asymptotics," IEEE Trans. Inf. Theory, vol. 51, pp. 2058-2072, Jun. 2005.

[28] L. Ahumada, R. Feick, R. A. Valenzuela, and C. Morales, "Measurement and characterization of the temporal behavior of fixed wireless links," IEEE Trans. Vech. Tech., vol. 54, pp. 1913-1922, Nov. 2005.

[29] T. S. Rappaport, Wireless Communications: Principles and Practice, 2nd ed. Prentice Hall, 2002.

[30] K. Liu, V. Raghavanand, and A. M. Sayeed, "Capacity scaling and spectral efficiency in wideband correlated MIMO channels," IEEE Trans. Inf. Theory, vol. 49, pp. 2504-2526, Oct. 2003.

[31] W. Hachem, Ph. Loubaton, and J. Najim, "Deterministic equivalents for certain functionals of large random matrices," Annals of Applied Probability, vol. 17, pp. 875-930, 2007.

[32] B. Furht and S. A. Ahson, Long Term Evolution: 3GPP LTE radio and cellular technology, Crc Press, 2009.

[33] R. Rajashekar and L. Hanzo, "Hybrid beamforming in mm-Wave MIMO systems having a finite input alphabet," IEEE Trans. Commun., vol. 64, pp. 3337-3349, Jun. 2016.

[34] T. M. Cover and J. A. Thomas, Elements of Information Theory, 2nd ed. New York: Wiely, 2006.

[35] Y. Wu, C.-K. Wen, C. Xiao, X. Gao, and R. Schober, "Linear precoding for the MIMO multiple access channel with finite alphabet inputs and statistical CSI,” IEEE Trans. Wireless. Commun., vol. 14, pp. 983-997, Feb. 2015.

[36] D. P. Palomar and S. Verdú, "Gradient of mutual information in linear vector Gaussian channels," IEEE Trans. Inf. Theory, vol. 52, pp. 141-154, Jan. 2006.

[37] Y. Wu, C. Xiao, Z. Ding, X. Gao, and S. Jin, "Linear precoding for finite alphabet signaling over MIMOME wiretap channels," IEEE Trans. Veh. Technol., vol. 61, pp. 2599-2612, Jul. 2012.

[38] Y. Wu, M. Wang, C. Xiao, Z. Ding, and X. Gao, "Linear precoding for MIMO broadcast channels with finite-alphabet constraints," IEEE Trans. Wireless Commun., vol. 11, pp. 2906-2920, Aug. 2012.

[39] Y. Wu, C. Xiao, X. Gao, J. D. Matyjas, and Z. Ding, "Linear precoder design for MIMO interference channels with finite-alphabet signaling," IEEE Trans. Commun., vol. 61, pp. 3766-3780, Sep. 2013.

[40] D. Tse and P. Viswanath, Fundamentals of Wireless Communication, New York, NY: Cambridge University Press, 2005. 
[41] F. Boccardi, R. W. Heath Jr., A. Lozano, T. L. Marzella, P. Popovski, "Five disruptive technology directions for 5G," IEEE Comm. Mag., vol. 52, pp. 74-80, Feb. 2014.

[42] M. Viberg, B. Ottersten, and A. Nehorai, "Performance analysis of direction finding with large arrays and finite data," IEEE Trans. Signal Process., vol. 43, pp. 469-477, Feb. 1995.

[43] F. Rusek, D. Persson, B. K. Lau, E. G. Larsson, T. L. Marzetta, O. Edfors, and F. Tufvesson, "Scaling up MIMO: Opportunities and challenges with very large arrays,” IEEE Signal Process. Mag., vol. 30, pp. 40-60, Jan. 2013.

[44] C. Sun, X. Gao, S. Jin, M. Matthaiou, Z. Ding, and C. Xiao, "Beam division multiple access transmission for massive MIMO communications," IEEE Trans. Commun., vol. 63, pp. 2170-2184, Jun. 2015.

[45] A. Adhikary, J. Nam, J-Y. Ahn, and G. Caire, "Joint spatial division and multiplexing-The large-scale array regime," IEEE Trans. Inf. Theory, vol. 59, pp. 3735-3750, Oct. 2013.

[46] H. Nishimori, Statistical physics of spin glasses and information processing: An introduction. Ser. Number 111 in Int. Series on Monographs on Physics. Oxford University Press, 2001.

[47] S. F. Edwards and P. W. Anderson, “Theory of spin glasses," J. of Physics F: Metal Physics, vol. 5, pp. 965-974, May 1975.

[48] T. Tanaka, "A statistical-mechanics approach to large-system analysis of CDMA multiuser detectors," IEEE Trans. Inf. Theory, vol. 48, pp. 2888-2910, Nov. 2002.

[49] A. L. Moustakas, S. H. Simon, and A. M. Sengupta, "MIMO capacity through correlated channels in the presence of correlated interferers and noise: A (not so) large $n$ analysis," IEEE Trans. Inf. Theory, vol. 49, pp. 2545-2561, Oct. 2003.

[50] D. Guo and S. Verdú, "Randomly spread CDMA: Asymptotics via statistical physics," IEEE Trans. Inf. Theory, vol. 51, no. 6, pp. 1983-2010, June 2005.

[51] C.-K. Wen and K.-K. Wong, "Asymptotic analysis of spatially correlated MIMO multiple-access channels with arbitrary signaling inputs for joint and separate decoding," IEEE Trans. Inf. Theory, vol. 53, pp. 252-268, Jan. 2007.

[52] R. Müller, D. Guo, and A. Moustakas, "Vector precoding for wireless MIMO systems and its replica analysis," IEEE J. Sel. Areas Commun., vol. 26, pp. 486-496, Apr. 2008.

[53] C.-K. Wen, S. Jin, and K.-K. Wong, "On the sum-rate of multiuser MIMO uplink channels with jointly-correlated Rician fading," IEEE Trans. Commun., vol. 59, pp. 2883-2895, Oct. 2011.

[54] T. K. Y. Lo, "Maximum ratio transmission,” IEEE Trans. Commun., vol. 47, pp. 1458-1461, Oct. 1999.

[55] B. M. Zaidel, R. R. Müller, A. L. Moustakas, and R. de Miguel, "Vector precoding for Gaussian MIMO broadcast channels: Impact of replica symmetry breaking," IEEE Trans. Inf. Theory, vol. 58, pp. 1413-1440, Mar. 2012.

[56] S. Boyd and L. Vandenberghe, Convex Optimization. New York: Cambridge University Press, 2004. 